\title{
Photometric scaling relations of anti-truncated stellar discs in SO-Scd galaxies ${ }^{\star}$
}

\author{
M. Carmen Eliche-Moral ${ }^{1}$, Alejandro Borlaff ${ }^{2}$, John E. Beckman ${ }^{2,3,4}$, and Leonel Gutiérrez ${ }^{5}$ \\ 1 Departamento de Astrofísica y CC. de la Atmósfera, Universidad Complutense de Madrid, 28040 Madrid, Spain \\ e-mail: mceliche@ucm.es \\ 2 Instituto de Astrofísica de Canarias, C/vía Láctea, 38200 La Laguna, Tenerife, Spain \\ 3 Departamento de Astrofísica, Universidad de La Laguna, 38200 La Laguna, Tenerife, Spain \\ ${ }^{4}$ Consejo Superior de Investigaciones Científicas, Spain \\ 5 Instituto de Astronomía, Universidad Nacional Autónoma de México, Ensenada BC 22860, Mexico
}

Received 28 July 2014 / Accepted 18 May 2015

\begin{abstract}
Context. It has been recently found that the characteristic photometric parameters of anti-truncated discs in S0 galaxies follow tight scaling relations.

Aims. We investigate whether similar scaling relations are satisfied by galaxies of other morphological types.

Methods. We have analysed the trends in several photometric planes relating the characteristic surface brightness and scalelengths of the breaks and the inner and outer discs of local anti-truncated S0-Scd galaxies, using published data and fits performed to the surface brightness profiles of two samples of Type-III galaxies in the $R$ and Spitzer $3.6 \mu \mathrm{m}$ bands. We have performed linear fits to the correlations followed by different galaxy types in each plane, as well as several statistical tests to determine their significance.

Results. We have found that 1) the anti-truncated discs of all galaxy types from Sa to Scd obey tight scaling relations both in $R$ and $3.6 \mu \mathrm{m}$, as observed in S0s;2) the majority of these correlations are significant accounting for the numbers of the available data samples; 3 ) the trends are clearly linear when the characteristic scalelengths are plotted on a logarithmic scale; and 4) the correlations relating the characteristic surface brightnesses of the inner and outer discs and the breaks with the various characteristic scalelengths significantly improve when the scalelengths are normalized to the optical radius of the galaxy. The observational uncertainties prevent us from discerning robustly whether the trends differ between the different types and bands, but we do not find statistical evidence of significant differences between the distributions of S0s and spirals or of barred and unbarred galaxies. These results suggest that the scaling relations of Type-III discs are independent of the morphological type and the presence (or absence) of bars within the observational uncertainties of the available datasets. However, larger and deeper samples are required to confirm this.

Conclusions. The tight structural coupling implied by these scaling relations imposes strong constraints on the mechanisms proposed to explain the formation of anti-truncated stellar discs in the galaxies across the whole Hubble sequence.
\end{abstract}

Key words. galaxies: elliptical and lenticular, cD - galaxies: spiral - galaxies: structure - galaxies: photometry - galaxies: evolution galaxies: fundamental parameters

\section{Introduction}

Erwin et al. (2005) introduced for the first time a definition of anti-truncated or Type-III galaxies as those in which the surface brightness of the disc does not follow the typical exponentially decaying profile with the radius (Patterson 1940; de Vaucouleurs 1957, 1958; Freeman 1970), but presents an up-bending profile, with the outer disc exhibiting a shallower slope than the inner disc outside a given radius (known as the break radius, $R_{\text {brkIII }}$ ). This nomenclature was an extension of the classification defined by Freeman (1970), who classified Type-I discs as those with single exponentially decaying profiles and Type-II discs as those with down-bending profiles outside the break radius (see also van der Kruit 1979, 1987).

In edge-on systems, anti-truncations tend to coincide with the superposition of a thin disc and a thick disc (Comerón et al. 2012). However, while nearly all Type-II profiles are associated with galaxy subcomponents (such as rings, pseudorings, lenses, or strong star formation regions), only $\sim 1 / 3$ of Type-III profiles

^ Tables 2-7 are available in electronic form at http: //www . aanda.org are related to distinct morphological substructures (see Laine et al. 2014, hereafter L14). The structural properties and frequencies of anti-truncations seem to differ in S0 and spiral types. The percentage of anti-truncations increases from $\sim 10-20 \%$ in Sc-Sd galaxies to $20-50 \%$ in S0-Sa types (Erwin et al. 2008; Ilyina \& Sil'chenko 2012; Gutiérrez et al. 2011, hereafter G11; L14; Maltby et al. 2015). In spirals, anti-truncations are basically disc-related phenomena, with less than $\sim 15 \%$ of them associated with the contribution of central spheroidal components to the galaxy outskirts (Maltby et al. 2012b, 2015). However, this percentage increases to $\sim 25 \%$ in $\mathrm{S} 0-\mathrm{Sb}$ galaxies (Erwin et al. 2005 ) and up to $\sim 50 \%$ if only SOs are considered (Maltby et al. 2015).

Type-II profiles are known to be related to bars in most cases (see e.g. Kim et al. 2014), but the origin of Type-III discs is still poorly constrained. Diverse mechanisms have been proposed to explain the formation of anti-truncations. The majority of them are related to gravitational or tidal interactions, such as minor mergers (Laurikainen \& Salo 2001; Peñarrubia et al. 2006; Younger et al. 2007), major mergers (Borlaff et al. 2014), interactions of the disc with dark matter subhaloes (Kazantzidis et al. 2009), high-eccentricity fly-by encounters (Younger et al. 2008), 
or harassment (Roediger et al. 2012). Other formation scenarios include the existence of different star formation thresholds as a function of the radius in the galaxy (Elmegreen \& Hunter 2006), ram-pressure stripping (Roediger et al. 2012), ongoing gas accretion (Minchev et al. 2012), and simple fading of stellar discs (Maltby et al. 2015). Herpich et al. (2015) have also proposed that the disc profile type of a galaxy may basically depend on the initial spin of its host halo. It seems that bars are unrelated to anti-truncations, as derived from the observational fact that the relative frequency of Type-III profiles found in samples of barred and unbarred galaxies is similar (Sil'chenko 2009; Erwin et al. 2008, hereafter E08; G11; L14). However, this needs to be confirmed by other means.

Recently, Borlaff et al. (2014) have found that the structures of the inner and outer discs and the location of the break in TypeIII S0 galaxies are strongly coupled, and this coupling seems to be independent of the existence of bars in the galaxies. These authors have shown that the characteristic photometric parameters of the inner and outer discs and the breaks in S0s satisfy several scaling relations, tighter in many cases if the scalelengths are normalized to the optical size of the galaxy. The question is whether or not these scaling relations (or similar ones) are also obeyed by Type-III discs of other morphological types. If not this would imply that anti-truncations do form through diverse and independent mechanisms in different Hubble types (which seems reasonable if we consider the wide variety of possible formation processes proposed for them). However, if the antitruncated discs of spiral galaxies satisfy scaling relations similar to those observed in S0s, it becomes challenging to understand the physical processes underlying this coupling in galaxies spanning the whole Hubble sequence. Analogously, if bars are relevant in determining the structure of some anti-truncated discs or have triggered their formation in some cases, we should expect to find significant differences between the photometric trends followed by Type-III discs of barred and unbarred galaxies, whereas negligible differences would be expected if both phenomena are structurally unrelated.

Therefore, we have investigated whether the Type-III discs of spirals obey scaling relations that are as tight as those observed in anti-truncated SOs and, in this case, whether the scaling relations can be considered similar or both galaxy types exhibit significant differences between them. The same analysis has been performed for barred and unbarred galaxies to find out whether bars and anti-truncations are structurally related.

For this purpose, we have used the data published by E08 and G11 in the $R$ band, and by L14 in the $3.6 \mu \mathrm{m}$ Spitzer band. In Sect 2, we briefly comment on the galaxy samples of these authors, their data, and the procedures they followed to obtain and characterize the surface brightness profiles. Section 3 describes our fitting technique to the trends found in the studied photometric planes, as well as the tests performed to identify the correlations that were statistically significant. In Sect. 4 we show the main trends and scaling relations that we have found involving the characteristic scalelengths of the inner and outer discs ( $h_{\mathrm{i}}$ and $h_{\mathrm{o}}$, respectively), $R_{\text {brkIII }}$, and $R_{25}$. In this section we also statistically analyse the differences and similarities of the trends followed by S0 vs. spiral galaxies, followed by barred vs. unbarred galaxies, and of $R$ vs. $3.6 \mu \mathrm{m}$ data. Finally, the discussion and main conclusions are provided in Sects. 5 and 6.

\section{Data}

We have analysed the possible correlations between the characteristic parameters of the breaks and the inner and outer discs of
Table 1. Statistics of Hubble types (S0 and spiral) and barred and unbarred galaxies for the $R$ and $3.6 \mu \mathrm{m}$ samples of Type-III galaxies.

\begin{tabular}{llll}
\hline \hline$R$ band $^{a}$ & & & \\
& Barred $^{b}$ & Unbarred $^{c}$ & Total \\
\hline S0-S0/a & $9(22.5 \%)$ & $12(30 \%)$ & $21(52.5 \%)$ \\
Sa-Sbc & $7(17.5 \%)$ & $12(30 \%)$ & $19(47.5 \%)$ \\
All Hubble types & $16(40 \%)$ & $24(60 \%)$ & $40(100 \%)$ \\
\hline $3.6 \mu$ band $^{a, d}$ & & & \\
& Barred & Unbarred & Total \\
\hline S0-S0/a & $13(21 \%)$ & $18(29 \%)$ & $31(50 \%)$ \\
Sa-Scd & $16(26 \%)$ & $15(24 \%)$ & $31(50 \%)$ \\
All Hubble types & $29(47 \%)$ & $33(53 \%)$ & $62(100 \%)$ \\
\hline
\end{tabular}

Notes. ${ }^{(a)}$ The percentages are given with respect to the total number of galaxies in the sample of each band. ${ }^{(b)}$ All barred galaxies in the $R$-band sample are from E08. ${ }^{\left({ }^{c}\right)}$ All unbarred galaxies in the $R$-band sample are from G11. ${ }^{(d)}$ The data in the $3.6 \mu \mathrm{m}$ band come from L14.

two samples of local galaxies with Type-III stellar discs, in the $R$ and $3.6 \mu \mathrm{m}$ bands. The $R$-band dataset contains the photometric parameters derived for 16 Type-III barred nearby galaxies by E08 and for 24 Type-III unbarred ones by G11 (40 in total), all with S0-Sbc types. The $3.6 \mu \mathrm{m}$ dataset comprises the 62 TypeIII (barred and unbarred) galaxies from the sample analysed by L14, with types spanning from S0 to Scd. Our study is exclusively centred on galaxies with pure Type-III profiles, i.e. the galaxies with hybrid profiles from the original samples (usually referred to as Type II+III) have been excluded in our subsamples ( 4 galaxies in the E08 sample, 5 from G11, and 7 from L14) to avoid a possible additional dispersion in the trends we were looking for. The E08 sample overlaps with the L14 sample in 4 galaxies, while G11 sample has 7 galaxies in common with L14.

Table 1 summarizes the statistics of the samples in terms of morphological types and the barred/unbarred nature in both bands. The statistics of the two subsamples is not very high (40 S0-Sbc galaxies in $R$ and 62 S0-Scd's in $3.6 \mu \mathrm{m}$ ), but the numbers are sufficiently large to allow us to look for photometric scaling relations in $\mathrm{S} 0$ s and spirals separately, because the galaxies distribute nearly equally between the two types in both bands. A similar argument holds for barred and unbarred galaxies. The original data, reduction, and methodology to characterize the surface brightness profiles are extensively described in the original papers, so we provide just a brief description here.

The original samples were defined using different selection criteria for the radial velocities, angular sizes, galactic latitudes, and morphologies of the galaxies. The galaxies in the $R$-band sample have distances $<30 \mathrm{Mpc}$ and those of the L14 sample lie at $<80 \mathrm{Mpc}$, but both datasets present $-18<M_{B}<-22 \mathrm{mag}$. E08 and G11 used data in the $r$ and $R$ bands obtained with different telescopes, with PSF FWHM $\sim 0.7^{\prime \prime}$ and limiting surface brightness $\mu_{\text {lim }} \sim 26-27 \mathrm{mag} \mathrm{arcsec}^{-2}$ in $R$ (Vega system). L14 combined data obtained in the $3.6 \mu \mathrm{m}$ IRAC band for SaSd galaxies of the $S^{4} \mathrm{G}$ survey $\left(F W H M \sim 1.7^{\prime \prime}\right.$, see Sheth et al. 2010) with $K_{\mathrm{s}}$-band images for S0-S0/a galaxies from the NIRSOS survey ( $F W H M \sim 0.7^{\prime \prime}$, see Laurikainen et al. 2011). In L14, the distances of the galaxies coming from the $S^{4} \mathrm{G}$ sample are $<40 \mathrm{Mpc}$, and $<80 \mathrm{Mpc}$ for those coming from NIRSOS.

L14 converted the $K$-band surface brightness profiles of the galaxies from the NIRSOS sample to $A B$ magnitudes in the $3.6 \mu \mathrm{m}$ band accounting for the colour differences and magnitude offsets derived for the 93 galaxies that the two surveys have in common. These authors computed total magnitudes in elliptical apertures tracing the $\mu=22.5 \mathrm{mag} \operatorname{arcsec}^{-2}$ isophote in $3.6 \mu \mathrm{m}$ 
in each galaxy. The median difference between the magnitudes obtained in the two surveys was derived, including a linear term to describe the dependence on colour. This conversion factor was then applied to the surface brightness profiles and total magnitudes of the NIRSOS data to transform them into $3.6 \mu \mathrm{m}$. The L14 data finally presented $\mu_{\text {lim }} \sim 26.4$ mag $\operatorname{arcsec}^{-2}$ for the SaScd galaxies and $\mu_{\mathrm{lim}} \sim 24.7 \mathrm{mag} \operatorname{arcsec}^{-2}$ for the S0-S0/a galaxies in $3.6 \mu \mathrm{m}$ (AB magnitudes).

Both data samples are analogous in terms of depth for the spiral types, but the $R$-band sample is at least $\sim 1$ mag deeper than the $3.6 \mu \mathrm{m}$ sample for the S0 galaxies. L14 compared the limiting surface brightness of their sample with that of the $V$-band sample by Maltby et al. (2012a), finding that $V-[3.6] \sim 1.5 \mathrm{mag}$ (AB system, see their Sect. 6). Considering that $V-R$ ranges $\sim 0.2-0.5$ in the discs of Sa-Sd galaxies (Möllenhoff 2004) and $(V-R)=0.5-0.65$ in those of S0s (Gregg 1989), we find that the limiting magnitudes of the $3.6 \mu \mathrm{m}$ sample by L14 roughly correspond in the $R$ band to $\mu_{\text {lim }} \sim 27.5$ for the spirals and $\mu_{\text {lim }} \sim 25.5$ for the S0s (Vega system). Here, we have considered that $V(\mathrm{AB})-V($ Vega $)=0.02$ (Blanton \& Roweis 2007). Assuming that $\mu_{\mathrm{lim}} \sim 26.5 \mathrm{mag} \operatorname{arcsec}^{-2}$ on average in the E08 and G11 samples, this means that the $3.6 \mu \mathrm{m}$ data sample is $\sim 1$ mag $\operatorname{arcsec}^{-2}$ deeper than the $R$-band sample for the spirals. On the other hand, the E08 and G11 samples are $\sim 1$ mag arcsec ${ }^{-2}$ deeper than the L14 sample for the S0s. However, some profiles in E08 and G11 achieve $\mu_{\text {lim }} \sim 28 \mathrm{mag} \operatorname{arcsec}^{-2}$. So, the $R$-band sample may be reaching similar depths to the L14 sample in some specific cases.

E08 and G11 used the morphological types available in the RC3 catalogue (de Vaucouleurs et al. 1991), based on the optical morphology of the galaxies, whereas the types in L14 were assigned according to the morphology in their $K$ or $3.6 \mu \mathrm{m}$ images (Laurikainen et al. 2011; Buta et al. 2015). E08 considered as barred galaxies those exhibiting strong bars (SB) or weak bars (SAB) according to the RC3 classification, but revised the classes according to their deep $R$-band images and rejected the galaxies without clear bars in them or involved in strong interactions. The barred/unbarred classification in L14 was, however, made on the basis of their deep $K$ and $3.6 \mu \mathrm{m}$ images from the NIRSOS and $\mathrm{S}^{4} \mathrm{G}$ surveys.

The surface brightness profiles were obtained by azimuthally averaging the light within ellipses fitted to the isophotes of the galaxies. The three studies (E08, G11, and L14) held the values of the centre, ellipticity, and position angle of isophotes fixed to the values of the outer discs in the fits.

E08 and L14 fitted the disc profiles using "brokenexponential" functions, which describe the inner and outer discs through two exponentially decaying profiles joined by a transition region, according to the expression

$I(r)=S I_{0} \exp \left[\frac{-r}{h_{\mathrm{i}}}\right]\left\{1+\exp \left[\alpha\left(r-R_{\mathrm{brkIII}}\right)\right]\right\}^{\frac{1}{\alpha}\left(\frac{1}{h_{\mathrm{i}}}-\frac{1}{h_{\mathrm{o}}}\right),}$

where $I_{0}$ represents the central intensity of the inner exponential section, $\alpha$ parameterizes the sharpness of the break, and $S$ is a scaling factor, given by

$S=\left[1+\exp \left(-\alpha R_{\text {brkIII }}\right)\right]^{\frac{1}{\alpha}\left(\frac{1}{h_{\mathrm{i}}}-\frac{1}{h_{\mathrm{o}}}\right)}$.

On the other hand, G11 performed independent exponential fits to the inner and outer discs ("piecewise fits"), defining $R_{\text {brkIII }}$ as the radius at which the fitted profiles cross. The surface brightness of the profile at $r=R_{\text {brkIII }}$ is defined as the break surface brightness $\left(\mu_{\text {brkIII }}\right)$. E08 showed that the two fitting procedures provide very similar results (within $5 \%$ for the characteristic scalelengths in the case of Type-III profiles). This allows the comparison of the characteristic parameters of the samples by E08 and G11 in the $R$ band.

Consequently, we have used the characteristic parameters of the breaks and the inner and outer discs of Type-III galaxies derived by E08, G11, and L14 to compare the trends of S0 and spiral types in several photometric planes in the $R$ and $3.6 \mu \mathrm{m}$ bands. We note that the magnitudes of the $R$-band data are the Vega system and in $\mathrm{AB}$ for the $3.6 \mu \mathrm{m}$ dataset.

\section{Fits and correlation tests}

We performed linear fits $\left(y=m x+C_{0}\right)$ to the trends followed in each photometric plane by all galaxies, by spirals and S0s independently, by types of spirals (Sa-Sab, Sb-Sbc, Sc-Scd), and by barred and unbarred galaxies. The trends of the types in each sample were fitted using ordinary least squares. The photometric parameters of the inner and outer discs characterized by E08, G11, and L14 had no errors assigned in their original papers, so no error weighting could be considered in the fits. In order to estimate realistic confidence intervals to the fitted regression coefficients, we adopted a bootstrapping method. We generated $n=10^{5}$ artificial data samples with the same size as the original one in each diagram with replacement. The final regression coefficients of each fit correspond to the median values of the probability distributions of each coefficient obtained with the $10^{5}$ results, in order to reduce the systematic bias introduced by outliers. The upper and lower errors considered for each coefficient are those enclosing $2.5 \%$ and $97.5 \%$ of the values in the corresponding probability distribution. The bootstrap distribution is closer to the real probability distribution of the coefficients than a simple Gaussian in general, so this method derives robust and conservative (asymmetric) confidence intervals for the regression coefficients, reducing the effects of possible outliers or high leverage points at the same time.

We tested the significance of each photometric trend using the Spearman rank correlation test, which measures whether two variables are monotonically related and what the level of correlation between them is, and it has the advantage of being non-parametric. Only those trends with an associated probability of random correlation below 5\% according to the test $\left(p_{\mathrm{S}}<0.05\right)$ are considered as statistically significant. The $3.6 \mu \mathrm{m}$ dataset presents higher statistics than the $R$-band sample, but the Spearman rank correlation test accounts for the number of data pairs yielding the trend to derive $p_{\mathrm{S}}$. Additionally, the Pearson coefficient $(\rho)$ has been used to determine the level of linear correlation of each trend.

The slope $(m)$ and $Y$-intercept $\left(C_{0}\right)$ of the linear fits performed to the different trends analysed in each photometric plane, their asymmetric error intervals, as well as the values of $p_{\mathrm{S}}, \rho$, and the number of available data pairs $\left(N_{\text {pairs }}\right)$ for each trend, are listed in Tables 2-7.

Figures 1-9 show the trends followed by Type-III galaxies in these photometric planes. We have overplotted the obtained linear fits only when they fulfil the Spearman rank correlation test at a $95 \%$ significance level, i.e. only if there is a significant correlation in the diagram. We note that, although there may be a significant correlation between two parameters according to the Spearman test, it does not have to be significantly linear. In fact, some trends are significant according to the test $\left(p_{\mathrm{S}}<0.05\right)$, but they exhibit low values of the Pearson coefficient $\rho$ (e.g. the $\mu_{\text {brkIII }}-R_{\text {brkIII }}$ trend in $3.6 \mu \mathrm{m}$ in Fig. 2). In these figures, we have written the results of the most relevant fits being compared at the top of each panel even when the correlations are not significant. 

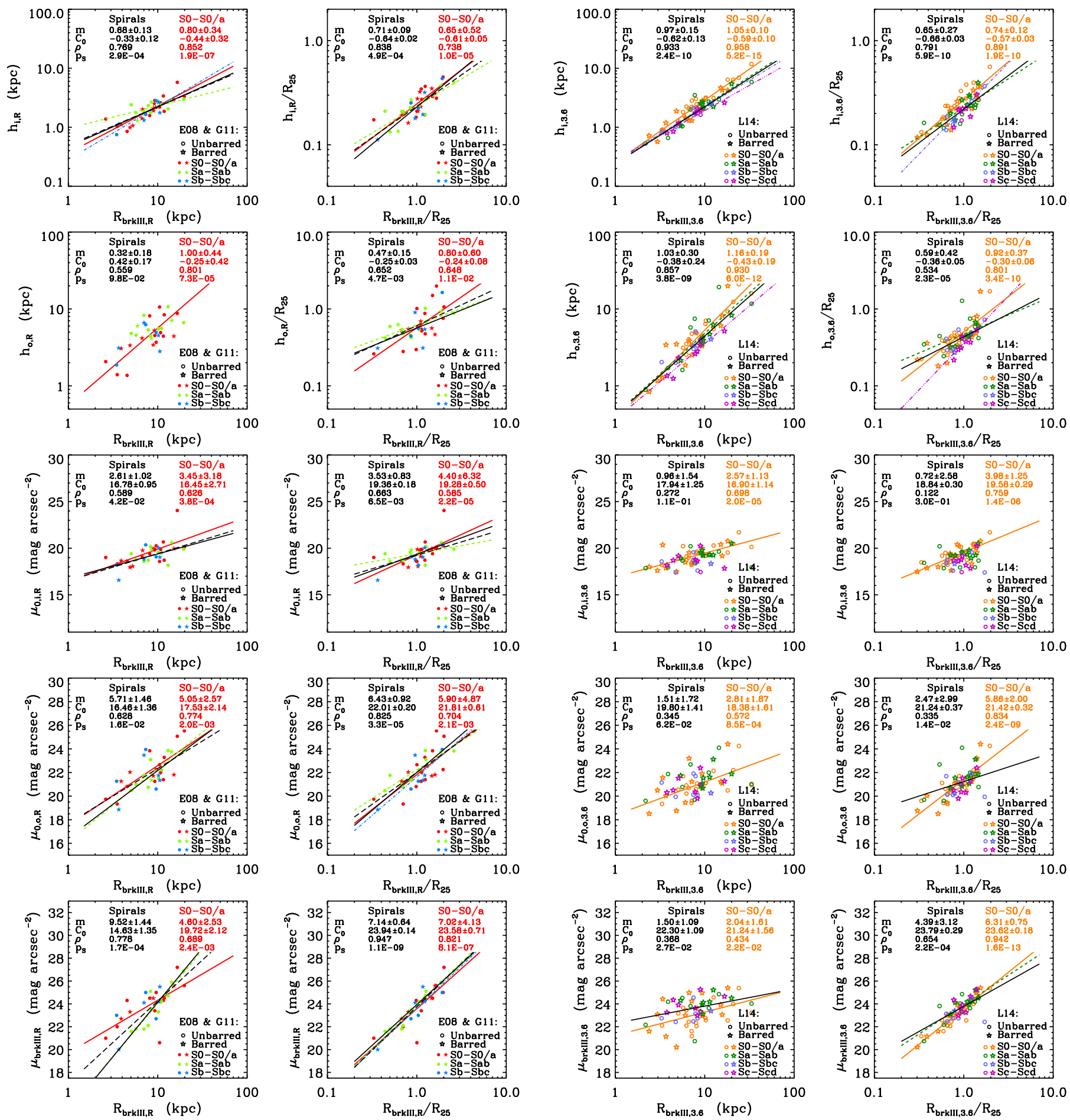

Fig. 1. Trends of the photometric parameters of the break and the inner and outer discs with $R_{\text {brkIII }}$ for the local anti-truncated S0-Sbc galaxies in the $R$ band from the E08 and G11 samples (see Tables 2-4).

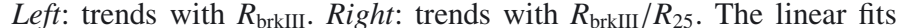
performed to each galaxy type are overplotted only if they are significant (red thick solid line: $\mathrm{S} 0-\mathrm{S} 0 / \mathrm{a}$, grey thin solid line: all spirals, green dashed line: Sa-Sab, blue dash-dotted line: $\mathrm{Sb}-\mathrm{Sbc}$ ). The results of the linear fits performed for the spirals and S0s are indicated at the top of each panel. The errors of the fits shown in the panels have been symmetrized for simplicity (the results are available in the corresponding tables). See the legend in the panels.

For simplicity, we have symmetrized the error interval of $m$ and $C_{0}$ in the figures, but the asymmetrical upper and lower errors

actually obtained for the coefficients of the fits are available in Tables 2-7. 
M. C. Eliche-Moral et al.: Scaling relations of anti-truncated stellar discs in S0-Scd galaxies
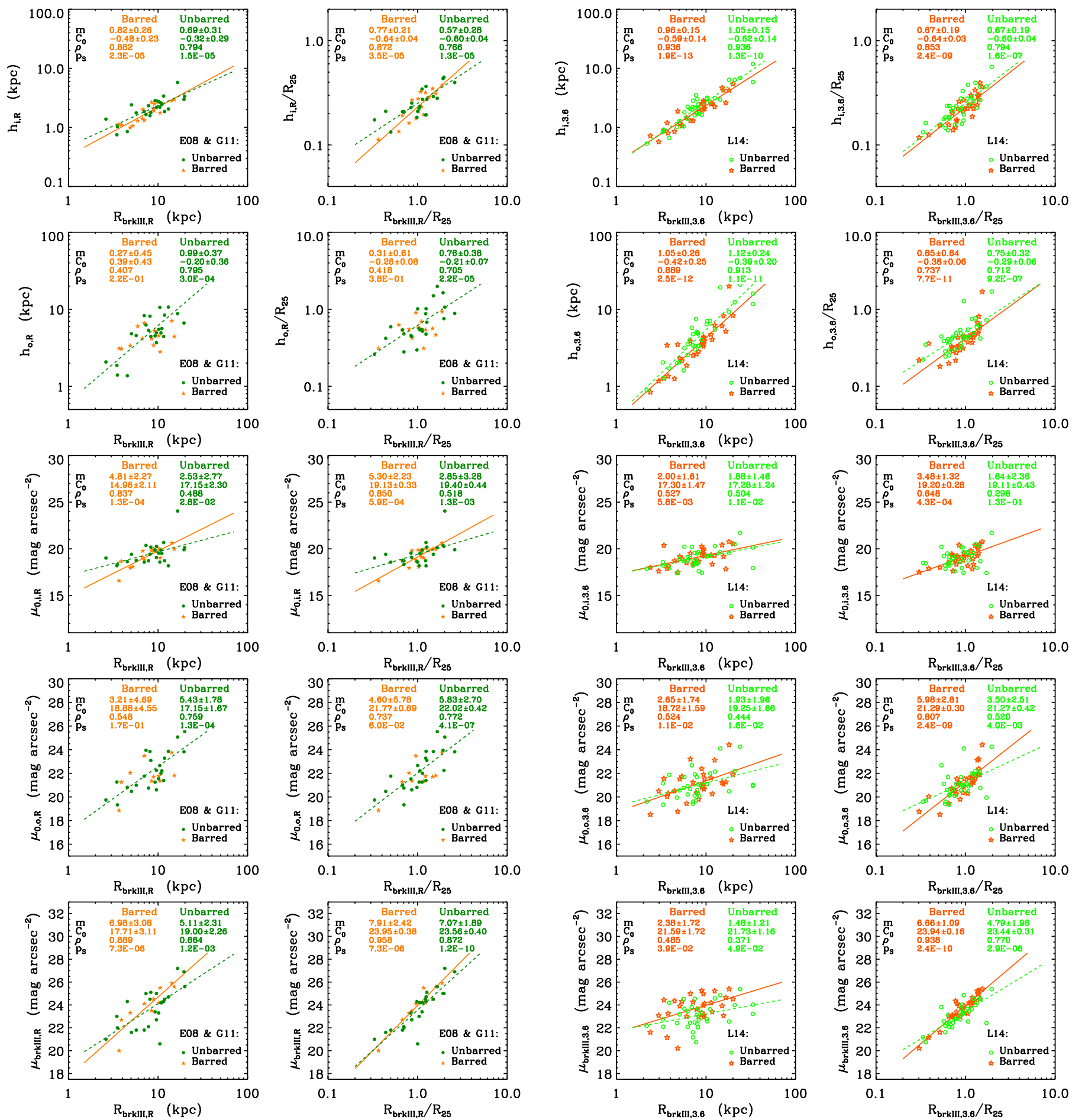

Fig. 3. Same as Fig. 1, but for barred and unbarred galaxies in the $R$ band (see Tables 2-4). The linear fits performed to barred and unbarred galaxies are overplotted only if they are significant (yellow solid line: barred galaxies, green dashed line: unbarred galaxies). The results of the linear fits are indicated at the top of each panel. The errors of the fits shown in the panels have been symmetrized for simplicity (the results are available in the corresponding tables). See the legend in the panels.

The characteristic scalelengths are plotted in logarithmic scales in all figures, because the correlations exhibit more defined linear trends in this way than when using linear scales. In many photometric planes, we have normalized the characteristic scalelengths $\left(h_{\mathrm{i}}, h_{\mathrm{o}}, R_{\text {brkIII }}\right)$ to the optical radius of each galaxy.
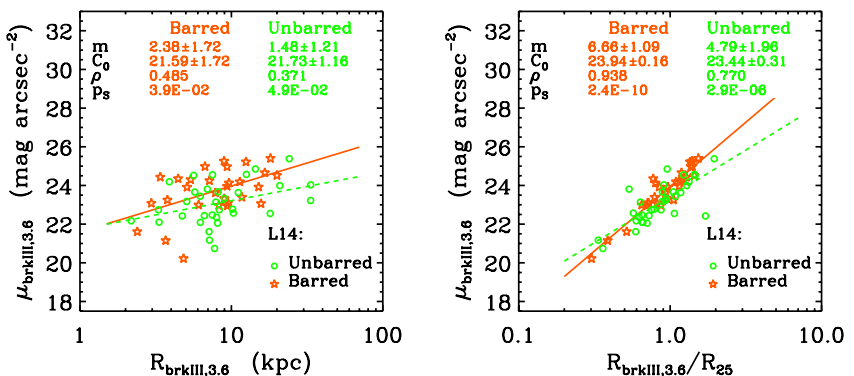

Fig. 4. Same as Fig. 2, but for local anti-truncated barred and unbarred galaxies in the $3.6 \mu \mathrm{m}$ band (Tables 2-4). See the caption of Fig. 3.

We have defined this following E08 and G11, i.e. as the radius of the isophote with $\mu=25 \mathrm{mag} \operatorname{arcsec}^{-2}$ in the $B$ band $\left(R_{25}\right)$. These authors provide $R_{25}$ for each galaxy in their samples, so we have used their tabulated values directly. The values of $R_{25}$ for the galaxies in the L14 sample were obtained from HyperLeda ${ }^{1}$, and include a correction for Galactic extinction and inclination effects.

1 The HyperLeda database is available at: http://leda. univ-lyon $1 . \mathrm{fr} /$ 

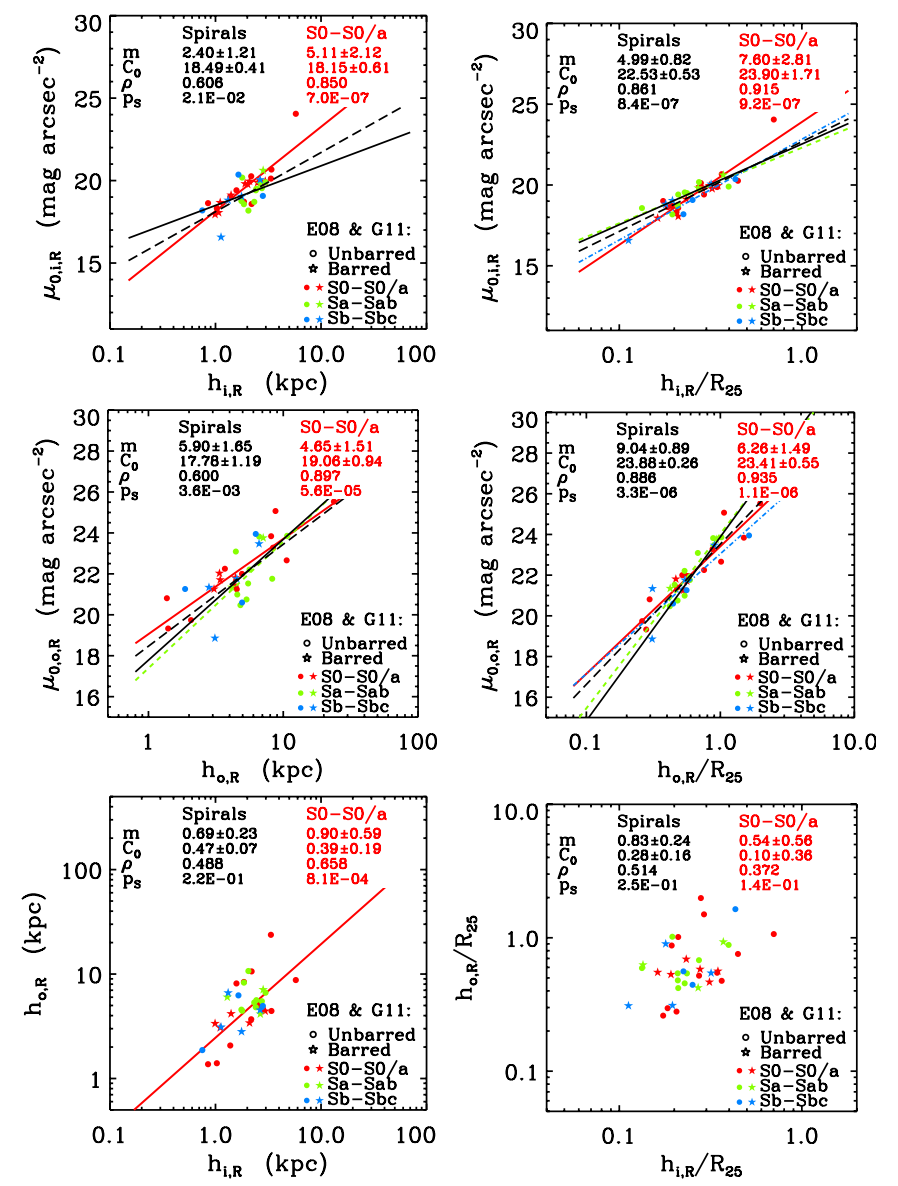

Fig. 5. Scaling relations between the parameters of the inner and outer discs of local anti-truncated $\mathrm{S} 0-\mathrm{Sbc}$ galaxies in the $R$ band from the E08 and G11 samples (results in Tables 5 and 6). See the caption of Fig. 1.

\section{Results}

In Sect. 4.1, we discuss the trends and correlations found in several photometric planes for the different morphological types and for barred and unbarred galaxies in the two datasets $(R$ and $3.6 \mu \mathrm{m})$. In Sect. 4.2, we compare the slopes and $Y$-intercepts of the linear trends fitted in each photometric plane for SOs and spirals, as well as for barred and unbarred. The fits obtained for the $R$-band and $3.6 \mu \mathrm{m}$ data are only compared in the photometric relations exclusively relating characteristic scalelengths.

\subsection{Trends and scaling relations}

\subsubsection{Trends with $R_{\text {brkllI }}$}

Figures 1 and 2 show the trends of several photometric parameters of the inner and outer discs of Type-III galaxies with $R_{\text {brkIII }}$ and $R_{\text {brkIII }} / R_{25}$ in the $R$ and $3.6 \mu \mathrm{m}$ bands, respectively. The two top rows of the figures display the trends of the inner and outer disc scalelengths with $R_{\text {brkIII }}$ by Hubble types in each band, in logarithmic scale. The distributions of spirals and S0s are similar in these planes and they overlap.

The main result is that $\log \left(h_{\mathrm{i}}\right), \log \left(h_{\mathrm{o}}\right), \mu_{0, \mathrm{i}}, \mu_{0, \mathrm{o}}$, and $\mu_{\text {brkIII }}$ correlate strongly with $\log \left(R_{\text {brkIII }}\right)$ in both spirals and S0s (all these trends have $\left.p_{\mathrm{S}}<0.05\right)$, and furthermore, similar correlations are obeyed for the different spiral types surveyed by each sample ( $\mathrm{Sa}-\mathrm{Sab}$ and $\mathrm{Sb}-\mathrm{Sbc}$ in both bands, and $\mathrm{Sc}-\mathrm{Scd}$ in $3.6 \mu \mathrm{m}$ ) within the observational uncertainties. The dispersions around the fitted linear trends in SOs and spirals are similar in
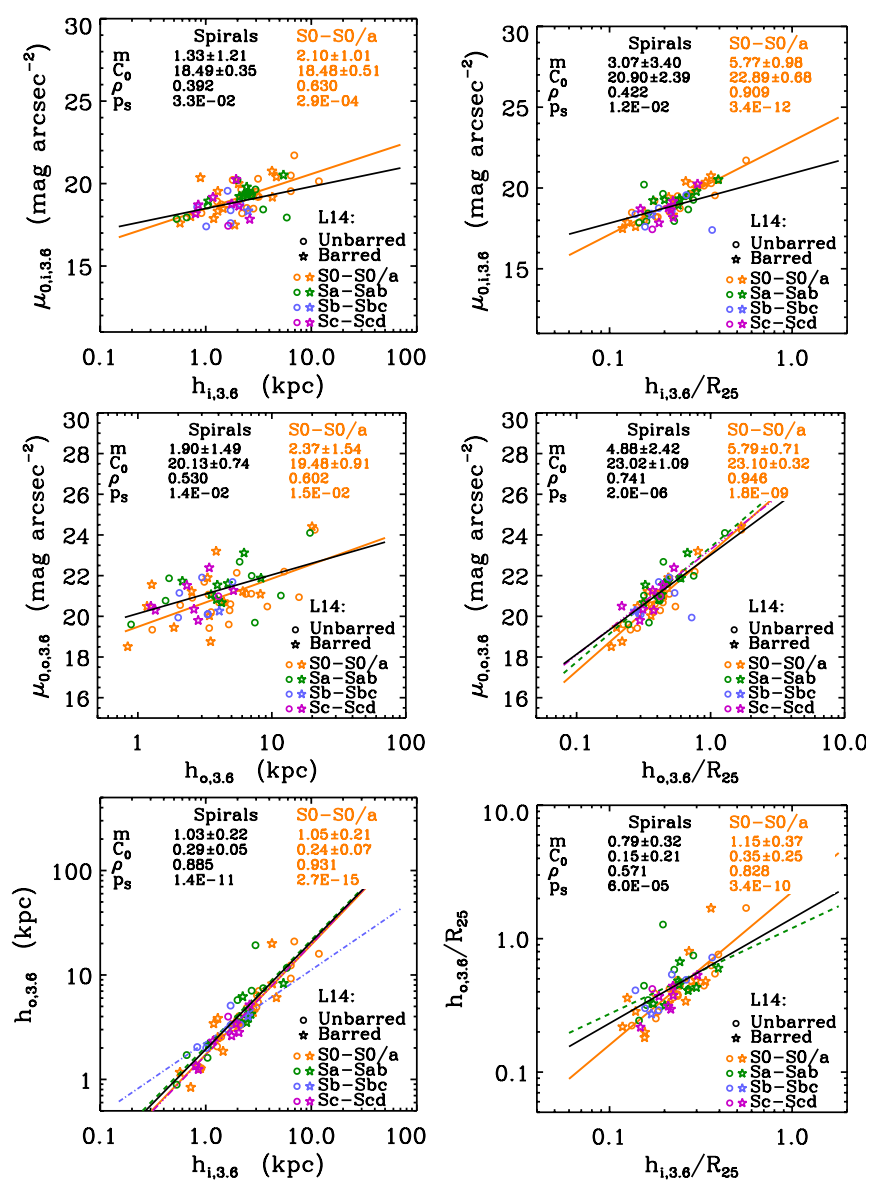

Fig. 6. Same as Fig. 5, but for local anti-truncated S0-Scd galaxies in the $3.6 \mu \mathrm{m}$ band from the L14 sample (results in Tables 5 and 6). See the caption of Fig. 2.

both bands, although the linear trends of $\log \left(h_{\mathrm{i}}\right)$ and $\log \left(h_{\mathrm{o}}\right)$ with $\log \left(R_{\text {brkIII }}\right)$ are better defined in $3.6 \mu \mathrm{m}$ than in $R$ (i.e. they have higher values of the linear correlation coefficient $\rho$ ), whereas it is the opposite in the trends involving $\mu_{0, i}, \mu_{0,0}$, and $\mu_{\text {brkIII }}$ (noticeable just by visual inspection of the trends).

The only diagrams in which spirals (globally or by types) show no significant correlation according to the Spearman rank correlation test are $h_{\mathrm{o}}-R_{\text {brkIII }}$ in $R$, and $\mu_{0, \mathrm{i}}$ or $\mu_{0, \mathrm{o}}$ vs. $R_{\text {brkIII }}$ (or $R_{\text {brkIII }} / R_{25}$ ) in $3.6 \mu \mathrm{m}$. The first may only be a question of the lower statistics of the $R$-band sample compared to the $3.6 \mu \mathrm{m}$ subsample, because the analogous plane in $3.6 \mu \mathrm{m}$ shows significant linear correlations for all spirals and by their sub-types, and the data distributions in both planes are quite similar. Correspondingly, the distributions of S0s and spirals in the diagrams of $\mu_{0, \mathrm{i}}\left(\right.$ or $\left.\mu_{0, \mathrm{o}}\right)-R_{\text {brkIII }}$ and $\mu_{0, \mathrm{i}}\left(\right.$ or $\left.\mu_{0, \mathrm{o}}\right)-R_{\text {brkIII }} / R_{25}$ in $3.6 \mu \mathrm{m}$ are also similar to the analogous distributions in the same diagrams of the $R$ band (compare the panels corresponding to $\mu_{0, \mathrm{i}}$ in both figures), so the lack of significance in the correlations in $3.6 \mu \mathrm{m}$ might also be a question of small numbers.

The trends of these photometric parameters with $\log \left(R_{\text {brkIII }} / R_{25}\right)$ present similar or even higher values of linear correlation (as measured by $\rho$ ) than with $\log \left(R_{\text {brkIII }}\right)$. In general, the correlations of $\mu_{0, \mathrm{i}}, \mu_{0, \mathrm{o}}$, and $\mu_{\mathrm{brkIII}}$ improve when $R_{\text {brkIII }}$ is normalized to the optical size of the galaxy (in particular, compare the trends and the Pearson coefficients of $\mu_{\text {brkIII }}$ $-R_{\text {brkIII }}$ and $\mu_{\text {brkIII }}-R_{\text {brkIII }} / R_{25}$ at the bottom panels of Figs. 1 and 2). The values of $\mu_{0, \mathrm{i}}, \mu_{0, \mathrm{o}}$, and $\mu_{\mathrm{brkII}}$ in Type-III discs are fainter as the breaks are more external (see the corresponding 
M. C. Eliche-Moral et al.: Scaling relations of anti-truncated stellar discs in S0-Scd galaxies
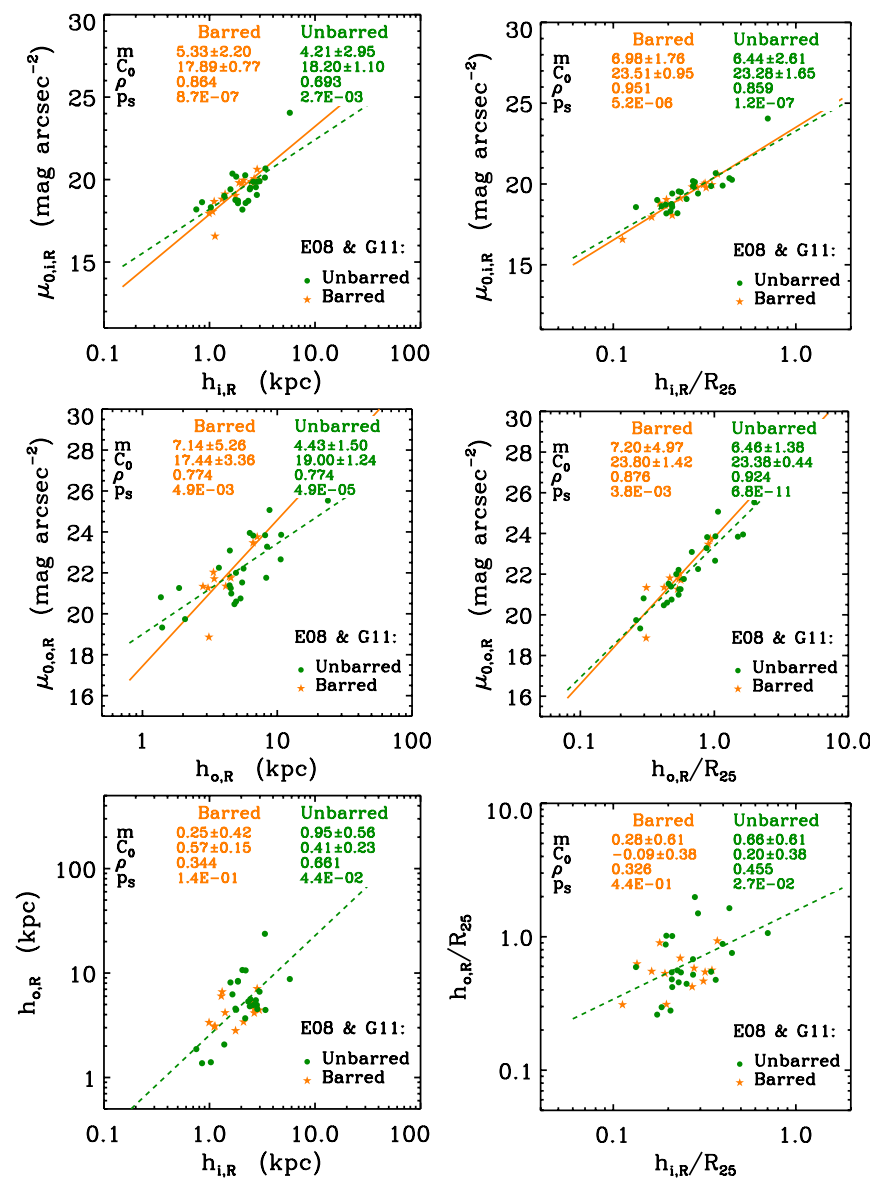

Fig. 7. Same as Fig. 5, but for barred and unbarred galaxies in the $R$ sample (the results are in Tables 5 and 6). See the caption of Fig. 3.

panels in the figures). However, these values seem to be more closely linked to the relative location of $R_{\text {brkIII }}$ with respect to the outer radius of the galaxy (as measured by $R_{25}$ ) than to $R_{\text {brkIII. }}$.

In Figs. 3 and 4 we plot the same photometric planes as in Figs. 1 and 2, but distinguishing between barred and unbarred galaxies. Again, the linear fits performed to the barred and unbarred galaxies have been overplotted only if they are significant. The majority of the photometric planes show significant scaling relations for both barred and unbarred galaxies in the two bands. The trends fitted to the barred galaxies look similar to those obtained for unbarred galaxies within the observational dispersion, as derived from the fact that the distributions for the two galaxy classes practically overlap in the diagrams. This suggests that bars seem to affect these scaling relations very little (at least, within the uncertainties implied by the data samples).

The only relations that are not significant in Figs. 3 and 4 are the trends involving $h_{\mathrm{o}}$ and $\mu_{0, \mathrm{o}}$ for barred galaxies in $R$ and the $\mu_{0, \mathrm{i}}-\log \left(R_{\mathrm{brkIII}} / R_{25}\right)$ trend in $3.6 \mu \mathrm{m}$ for the unbarred galaxies. But again, the lack of correlation in each band may reflect the low statistics of the samples.

The linear trends for barred and unbarred galaxies are very well defined in the $3.6 \mu \mathrm{m}$ dataset in the planes involving $h_{\mathrm{i}}$ and $h_{\mathrm{o}}$ while those relating $\mu_{0, \mathrm{i}}, \mu_{0, \mathrm{o}}$, and $\mu_{\text {brkIII }}$ with $\log \left(R_{\text {brkIII }}\right)$ have higher $\rho$ values in the $R$ band (as also happened in Figs. 1 and 2 for S0s and spirals). In any case, the trends in the photometric planes described by the $R$-band dataset look similar to their $3.6 \mu \mathrm{m}$ analogs taking into account the data dispersion. Again, we find that the linear correlation coefficients of the trends
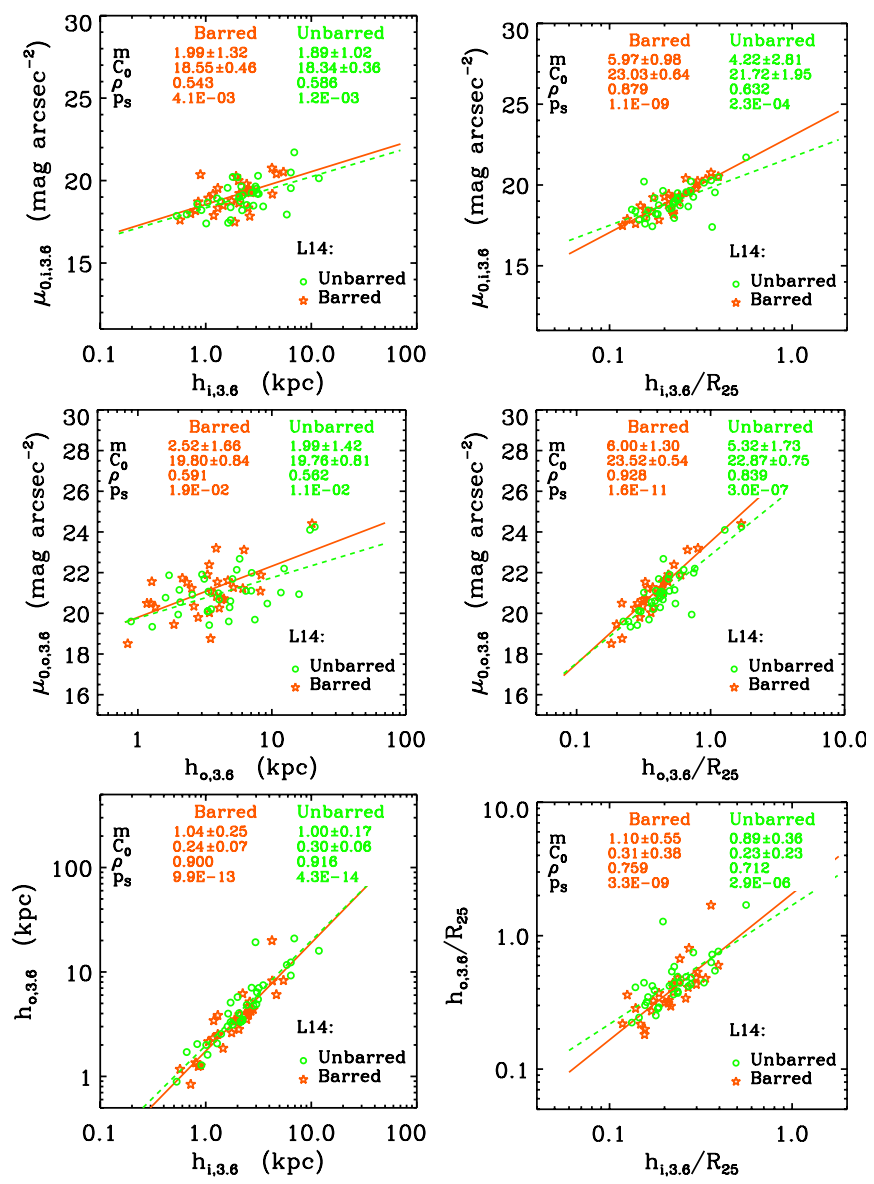

Fig. 8. Same as Fig. 6, but for barred and unbarred galaxies in the $3.6 \mu \mathrm{m}$ sample (the results are in Tables 5 and 6). See the caption of Fig. 4.

relating $\mu_{0, \mathrm{i}}, \mu_{0, \mathrm{o}}$, and $\mu_{\mathrm{brkIII}}$ with $\log \left(R_{\mathrm{brkIII}}\right)$ tend to improve if $R_{\text {brkIII }}$ is normalized to $R_{25}$, for both barred and unbarred galaxies (see the three rows of panels at the bottom of Figs. 3 and 4 ).

Summarizing, we have found that the inner and outer discs of anti-truncated spirals obey tight photometric scaling relations with $R_{\text {brkIII }}$, as Borlaff et al. (2014) discovered for Type-III S0 galaxies. The trends for each type look similar among different morphological types and among barred and unbarred galaxies within the dispersion of the data in the planes. This result suggests that anti-truncations and bars are structurally independent phenomena in galaxies.

\subsubsection{Trends with $h_{i}$ and $h_{0}$}

In Figs. 5 and 6 we analyse the basic scaling relations obeyed by the inner and outer discs of Type-III galaxies in $R$ and $3.6 \mu \mathrm{m}$, respectively. We also show the photometric trends with $h_{\mathrm{i}}$ and $h_{\mathrm{o}}$ normalizing these scalelengths by $R_{25}$. The different morphological types (S0s and spirals, as well as Hubble types) yield significant linear relations in these photometric planes too, again similar among them within the observed data dispersion. The distribution in the planes of spirals and S0s also overlap in these diagrams.

L14 already reported that the two exponential sections of galaxy discs of Type-II and Type-III in their sample independently satisfied the basic scaling relation observed in pure exponential discs between their central surface brightness and their scalelengths, although they did not distinguish between different Hubble types in their Fig. 11. The two top panels in the first 

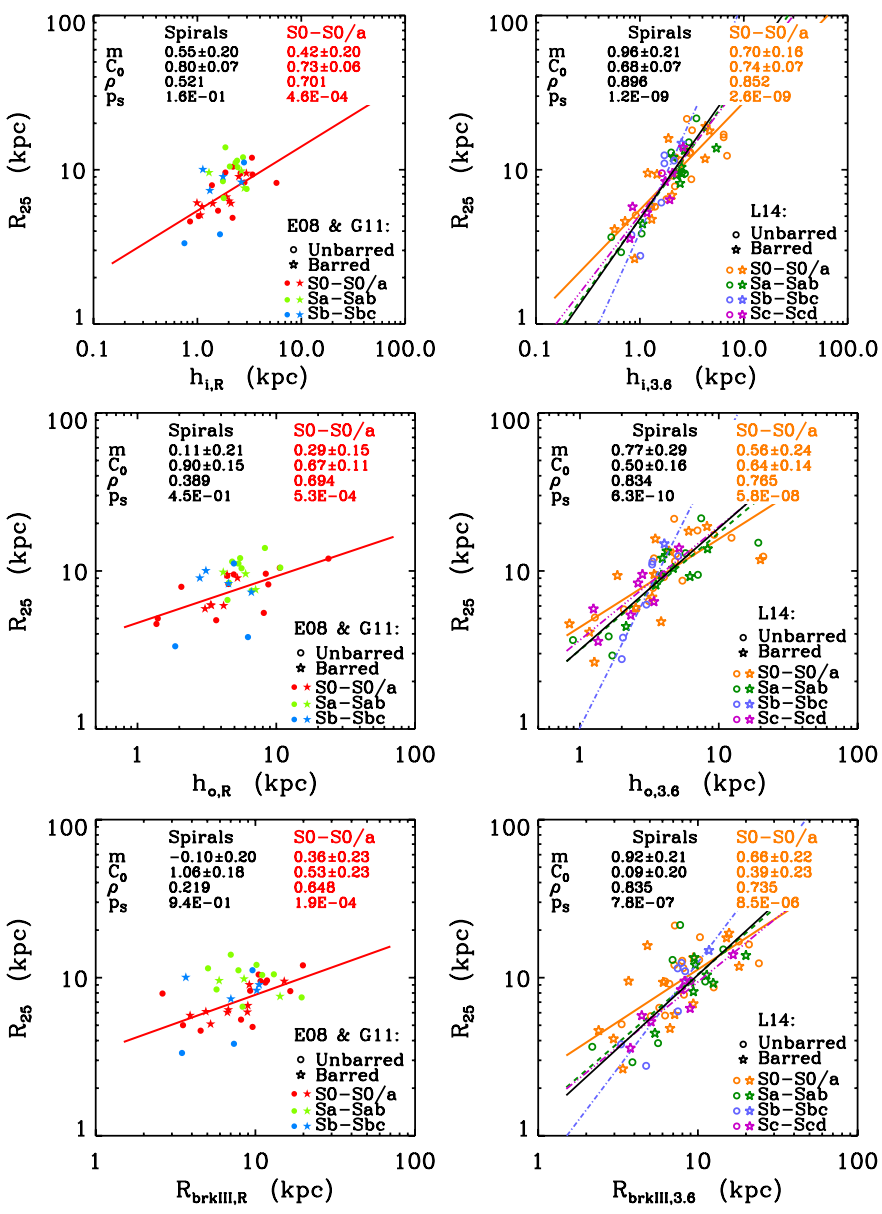

Fig. 9. Trends of $R_{25}$ with $h_{\mathrm{i}}, h_{\mathrm{o}}$, and $R_{\text {brkIII }}$ in local anti-truncated S0Scd galaxies in the $R$ and $3.6 \mu \mathrm{m}$ samples (left and right panels, respectively). The results are given in Table 7. See the captions of Figs. 1 and 2.

column of Fig. 6 show that this result also applies for different morphological types (S0, Sa-Sab, Sb-Sbc, and Sc-Scd) and spirals in general, and that it can be extended to the $R$ band (see the corresponding panels in Fig. 5).

Again, the linear correlations involving $\mu_{0, \mathrm{i}}$ and $\mu_{0, \mathrm{o}}$ improve noticeably when the disc scalelengths are normalized to $R_{25}$ for the two main galaxy types being considered (compare the left and right panels in the two figures). This is more striking in the $3.6 \mu \mathrm{m}$ trends, where this improvement can be noticed by visual inspection: the dispersion around the fitted linear trends in the $\mu_{0, \mathrm{i}}-h_{\mathrm{i}}$ and $\mu_{0, \mathrm{o}}-h_{\mathrm{o}}$ plots is significantly reduced in Fig. 6 when $h_{\mathrm{i}}$ and $h_{\mathrm{o}}$ are normalized to $R_{25}$. Moreover, $\rho$ increases significantly for both S0s and spirals in the two planes after this normalization.

The bottom panels of Fig. 6 show that $\log \left(h_{\mathrm{i}}\right)$ and $\log \left(h_{\mathrm{o}}\right)$ correlate linearly in both S0s and spiral galaxies in the $3.6 \mu \mathrm{m}$ band and in fact, this applies independently for $\mathrm{Sa}-\mathrm{Sab}, \mathrm{Sb}-\mathrm{Sbc}$, and $\mathrm{Sc}-\mathrm{Scd}$ types. In contrast, no significant trends are found in $R$, except for the S0s (see the same panel in Fig. 5). We note that the $\log \left(h_{\mathrm{o}}\right)-\log \left(h_{\mathrm{i}}\right)$ trends in $3.6 \mu \mathrm{m}$ do not improve if the scalelengths are normalized to $R_{25}$ (compare the bottom panels of Fig. 6).

We have plotted the same photometric relations in Figs. 7 and 8 , but now differentiating barred from unbarred galaxies. The linear fits obtained for each galaxy class (barred vs. unbarred) have been overplotted only if the correlations were significant according to the Spearman rank correlation test, as above. The figures show that barred and unbarred galaxies overlap in these diagrams and follow tight scaling relations in them, similar within the observational dispersion. Therefore, these scaling relations seem to be independent of the existence of a bar in the galaxy within the observational uncertainties, again suggesting that bars and anti-truncations are structurally unrelated phenomena.

In conclusion, we have found that the inner and outer discs of Type-III spirals obey tight scaling relations too, as observed in Type-III S0 galaxies. Again, we find that the existence of bars in the galaxies negligibly affect these scaling relations within the observational uncertainties and that the relations in the $\mu_{0, \mathrm{i}}-h_{\mathrm{i}}$ and $\mu_{0, \mathrm{o}}-h_{\mathrm{o}}$ planes significantly improve when the scalelengths are normalized by $R_{25}$.

\subsubsection{Trends with $R_{25}$}

As shown above, the linear correlations between the characteristic surface brightness values and the scalelengths become better defined in many photometric planes after normalizing the relevant parameters to $R_{25}$. We have analysed the trends between these characteristic scalelengths $\left(h_{\mathrm{i}}, h_{\mathrm{o}}\right.$, and $\left.R_{\text {brkIII }}\right)$ and $R_{25}$ in Fig. 9 for several galaxy types. First, the values of $R_{\text {brkIII }}, h_{\mathrm{i}}$, and $h_{\mathrm{o}}$ span similar ranges for a given $R_{25}$ in both $R$ and $3.6 \mu \mathrm{m}$, implying that both bands must be sampling the same type of breaks, but in different wavelength ranges.

In $R$, only the S0s exhibit significant trends of $\log \left(h_{\mathrm{i}}\right)$, $\log \left(h_{\mathrm{o}}\right)$, or $\log \left(R_{\text {brkIII }}\right)$ with $\log \left(R_{25}\right)$, even though S0s and spirals basically overlap in all planes (left panels in the figure). However, the $3.6 \mu \mathrm{m}$ dataset shows well-defined linear correlations for S0s and spirals, as well as for spiral subtypes in these diagrams (right panels). The trends look similar among them, as observed in the photometric parameters analysed previously. Again, the distributions of the $R$-band and $3.6 \mu \mathrm{m}$ data are similar in the same photometric planes, so the lack of correlations for spirals in $R$ might be due to the small numbers in the sample, as commented above.

The scaling relations in the right panels of Fig. 9 relate the size of the galaxy computed from an optical blue band $(B)$ with the structure of the inner and outer discs observed in a NIR band $(3.6 \mu \mathrm{m})$, indicating that there is a clear size scaling in these galaxies, such that larger (Type-III) discs have larger inner and outer disc scalelengths, and hence larger break radii. These scaling relations may present higher dispersion because $R_{25}$ is measured in the $B$ band by definition, a band that is certainly not a proxy of the galaxy stellar mass, whereas $h_{\mathrm{i}}, h_{\mathrm{o}}$, and $R_{\text {brkIII }}$ have been derived using data in much redder bands. However, the effects of dust in the $B$ band must be very limited at radial locations near $R_{25}$ in the discs, so we can assume that in practice the $B$ band does trace the stellar mass similarly to the $R$ or $3.6 \mu \mathrm{m}$ bands at these external radii. Moreover, the systematic improvement that we have found in many scaling relations after normalizing the scalelengths by $R_{25}$ implies that it must provide a robust estimate of the size of the stellar distribution, despite being computed in a blue band.

\subsection{Comparison of the trends}

In Sect. 4.1 we have seen that the scaling relations followed by S0s and spirals and by barred and unbarred galaxies look similar, both in the $R$ and $3.6 \mu \mathrm{m}$ bands. Here we analyse if there is 

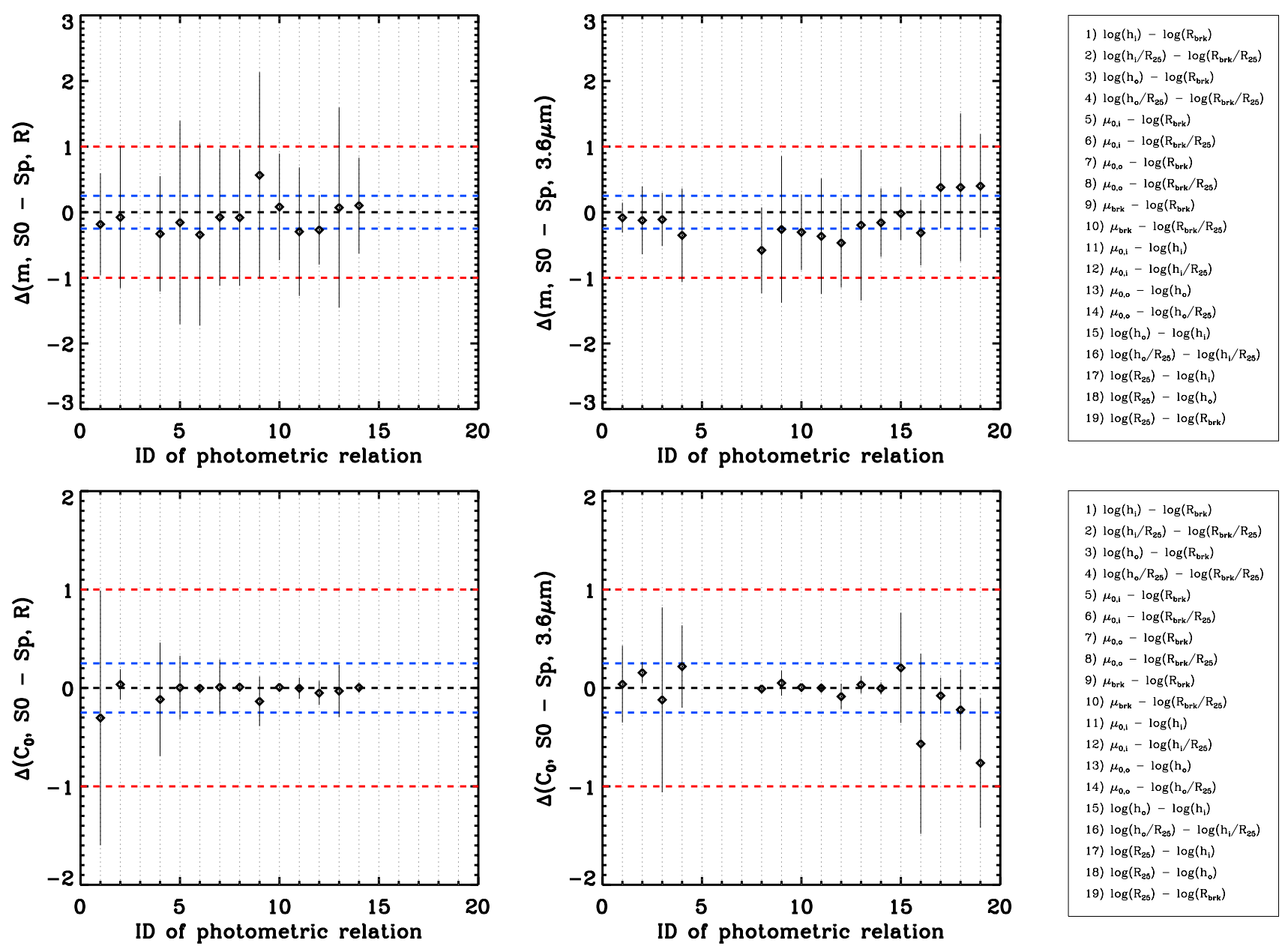

Fig. 10. Comparison of the relative differences of slope $(m)$ and $Y$-intercept $\left(C_{0}\right)$ values obtained from the linear fits performed to Type-III spirals vs. those performed to Type-III S0 galaxies in the photometric planes of Figs. 1-9. Left panels: $\Delta(m)$ and $\Delta\left(C_{0}\right)$ for the R-band data. Right panels: $\Delta(m)$ and $\Delta\left(C_{0}\right)$ for the $3.6 \mu \mathrm{m}$ data. Only the linear fits of the photometric trends that are significant according to the Spearman rank correlation test in the two datasets being compared are plotted. Blue and red horizontal dashed lines: limiting values such that the differences in $m$ and $C_{0}$ fitted to the S0s and spirals reach $25 \%$ and $100 \%$ of their values, respectively. See the key on the right for the numbers in the $X$-axis representing each photometric relation. Their numerical identifiers are the same as those used in Tables $2-7$.

significant statistical evidence that these relations differ within the observational uncertainties.

To this end we have considered the relative differences between the fitted values of the slopes and $Y$-intercepts for each trend in the two pair of datasets compared in each case (SO spirals, barred - unbarred). We define the relative difference of the slopes $m$ obtained for one photometric relation $i$ between S0s and spirals in a given band as follows:

$\Delta(m, \mathrm{~S} 0-\mathrm{Sp}$, band $)=\frac{m(\mathrm{Sp}, \text { band })-m(\mathrm{~S} 0, \text { band })}{m(\mathrm{~S} 0, \text { band })}$.

The errors in $\Delta(m, \mathrm{~S} 0-\mathrm{Sp}$, band $)$ correspond to the error propagation of the expression above, assuming as the error of each parameter the maximum between the absolute values of its upper and lower errors. Analogously, we have also defined the relative differences of the $Y$-intercepts $\left(C_{0}\right)$ for the trends fulfilled by two datasets being compared, $\Delta\left(\mathrm{C}_{0}, \mathrm{~S} 0-\mathrm{Sp}\right.$, band $)$, and their associated errors. These $\Delta$ values for $m$ and $C_{0}$ have only been defined when the two data samples being compared exhibit statistically significant correlations in the photometric relation separately, according to the Spearman rank correlation test.

Even if $\Delta(m)$ and $\Delta\left(C_{0}\right)$ in a given trend were nearly zero, this does not ensure that the trends can be considered similar, because it depends on their errors. However, if $\Delta(m) \sim 0$ and $\Delta\left(C_{0}\right) \sim 0$ with errors below a given (low) percentage, the trends of the two samples can be considered similar within these uncertainties. Obviously, we must keep in mind that deeper data can reveal differences in these trends that cannot be discriminated with the available datasets.

In Fig. 10, we compare the relative differences of the slopes and $Y$-intercepts of the linear fits performed to the SOs and the spirals for each of the 19 photometric relations analysed in Figs. 1-9, in $R$ and $3.6 \mu \mathrm{m}$ (left and right panels, respectively). We assume that two fitted linear trends can be considered similar if the differences in $m$ and $C_{0}$ and their errors are below $25 \%$. The figure shows that, under this criterion, no linear trend followed by S0s can be considered similar to the analogous one in spirals, either in the $R$ band or $3.6 \mu \mathrm{m}$. Although $|\Delta(m)|$ and $\left|\Delta\left(C_{0}\right)\right|$ are lower than 0.25 in many trends in each band (i.e. their values are contained within the horizontal blue lines in the planes of Fig. 10), their errors (in one case and/or another) exceed this limit. The statistics of the samples are too small to conclude that the trends are similar within some reasonable uncertainty level.

We have repeated the plot in Fig. 11, but comparing $\Delta(m)$ and $\Delta\left(C_{0}\right)$ for the linear trends followed by barred and unbarred galaxies. Again, only those trends that are significant in both 

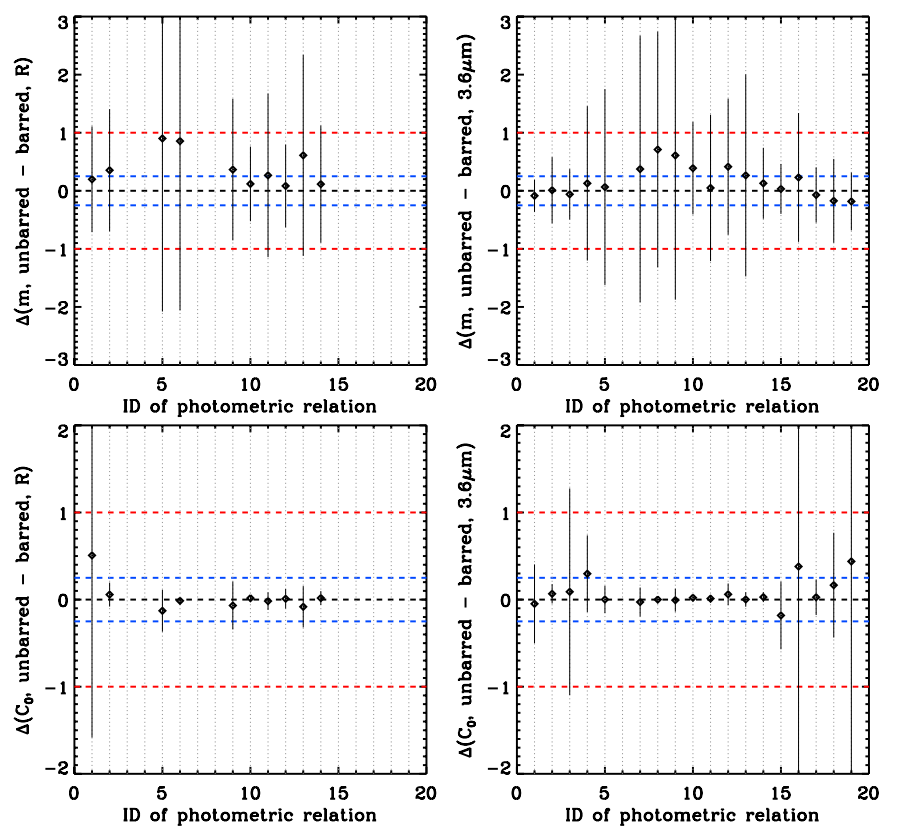

Fig. 11. Comparison of the relative differences of slope $(m)$ and $Y$-intercept $\left(C_{0}\right)$ values obtained from the linear fits performed to the barred galaxies vs. those performed to the unbarred galaxies in the photometric planes of Type-III galaxies being studied. Left: trends for the $R$-band data by E08 and G11. Right: trends for the $3.6 \mu \mathrm{m}$ data by L14. See the caption and legend of Fig. 10.

datasets are compared. The figure shows that the errors in these relative differences are too high again to conclude that the linear trends fitted to Type-III barred galaxies are similar to those of unbarred galaxies within uncertainties of $25 \%$, both in $R$ and $3.6 \mu \mathrm{m}$ (left and right panels, respectively).

The comparison of the trends between distinct bands involving physical scalelengths is also reasonable, because if the breaks correspond to a change in the projected stellar density, they should be observed at a similar physical location in the disc in several bands. Therefore, we have compared the linear fits performed to the trends relating two characteristic scalelengths in the $R$ and $3.6 \mu \mathrm{m}$ bands in Fig. 12. We have again considered only the trends that are significant according to the Spearman test in both bands. The left panels of the figure compare $\Delta(m)$ and $\Delta\left(C_{0}\right)$ for the linear trends fitted to the SOs in the two bands, while the right ones show the same for those fitted to spirals. Although the relative differences of $m$ and $C_{0}$ can be below $25 \%$ for many trends, the uncertainties are too high to assess whether these scaling relations observed in $R$ and $3.6 \mu \mathrm{m}$ are similar.

In summary, Figs. 10-12 show that the observational dispersion is too high to robustly discern whether the analysed scaling relations are similar in both S0s and spirals, in barred and unbarred galaxies, and in the $R$ and $3.6 \mu \mathrm{m}$ bands, although we do not find either statistical evidence of significant differences between the compared samples. Deeper data and larger samples are thus required to robustly confirm whether these scaling relations of anti-truncated discs are really independent of the morphological type and the presence (or absence) of bars.

\section{Discussion}

In Sect.4, we have shown that Type-III discs obey tight photometric scaling relations for galaxy types spanning the whole Hubble sequence. We have found no statistical evidence of noticeable differences between the relations followed by SOs and
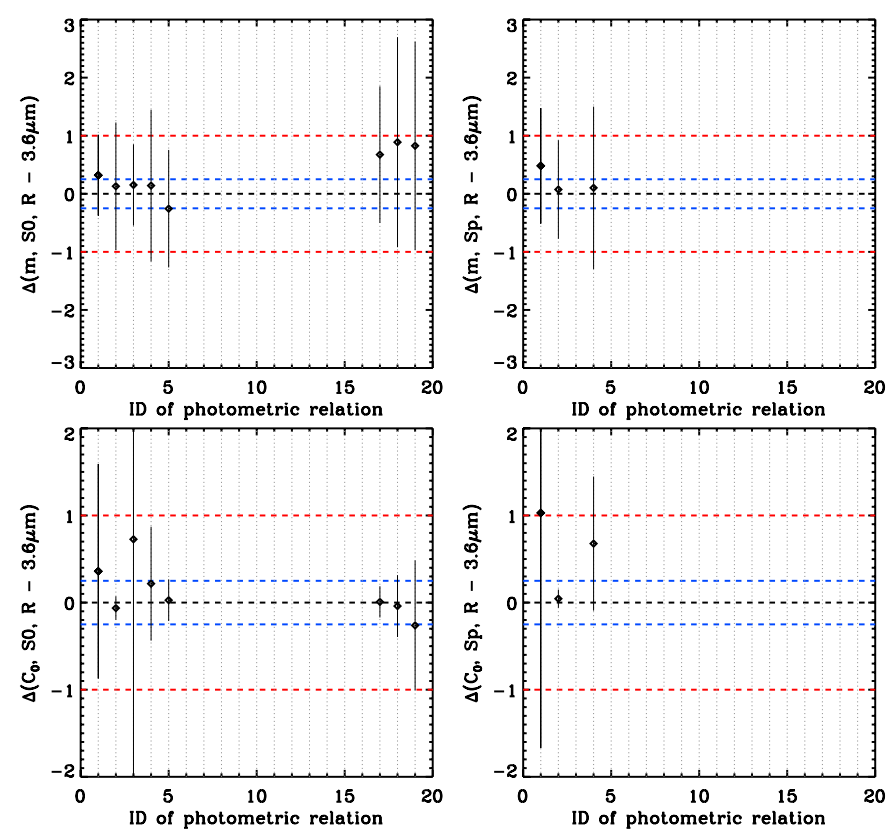

Fig. 12. Comparison of the relative differences of slope $(m)$ and $Y$-intercept $\left(C_{0}\right)$ values obtained from the linear fits performed to the $3.6 \mu \mathrm{m}$ data by L14 vs. those obtained from the $R$-band data by E08 and G11 in the photometric planes relating physical scalelengths under study. Left: trends for the S0 galaxies. Right: trends for spirals. See the caption and legend of Fig. 10.

spirals and barred and unbarred galaxies, a fact that suggests that the structure of anti-truncated discs may be independent of the galaxy type and the existence, or not, of bars in the galaxy. Although the statistical evidence is not strong considering the low numbers and the uncertainties of the available data samples, the structural independence of bars and anti-truncations agrees quite well with the similar relative frequency of Type-III profiles found in samples of barred and unbarred galaxies (E08; Sil'chenko 2009, G11; L14). Our results thus support the idea that bars and anti-truncations are decoupled structures in all morphological types. This does not imply that the two structures have formed independently, as some mechanisms are known to trigger the formation of both kinds of features, such as mergers or flybys (Walker et al. 1996; Lang et al. 2014). However, this is indicative that bars cannot have induced the formation of Type-III discs, as opposed to their tight structural link with Type-II discs (see e.g. Kim et al. 2014).

The scaling relations of Type-III discs found in the present study impose strong constraints on any formation scenarios proposed to explain the formation of anti-truncations, independently of whether the relations really depend on the morphological type or the hosting of a bar. Accounting for the wide diversity of mechanisms proposed to explain the formation of antitruncations (see Sect. 1), it is challenging to understand the physics underlying the scaling relations that we have found between Type-III discs across the whole Hubble sequence.

The dependence of these features on the environment becomes a key to discriminating between these mechanisms. Many studies have reported traces of recent or ongoing interactions in the outskirts of many Type-III discs, supporting a merging- or interaction-related nature (Erwin et al. 2005). L14 found a positive correlation between the scalelengths of Type-III discs and the tidal interaction strength, also pointing to external mechanisms. Coherently, flat and/or positive age gradients prevail in galaxies of the three profile types (in particular of Type-III) in 
the Virgo cluster, contrary to the expectations of scenarios in which the formation of these discs were mostly driven by secular inside-out disc growth and/or stellar migrations (Roediger et al. 2012). So, all these results suggest external processes as the main drivers of the formation of anti-truncations, probably as a result of the gravitational response of the disc to a tidal interaction.

However, in this case we should also expect to find some dependence of the structural properties of Type-III discs on the local galaxy density. On the contrary, however, the inner and outer disc scalelengths and the break strength ${ }^{2}$ of Type-III discs show no trends with the environment, either in spiral or S0 galaxies (Maltby et al. 2012a, 2015). Additionally, similar fractions of Type-III S0 galaxies are found in both cluster and field environments, suggesting that the environment hardly affects the outer structure of these galaxies (Erwin et al. 2012; Roediger et al. 2012). How can all these results be reconciled?

External processes may induce the formation of antitruncations as the result of gravitational-driven instabilities in the disc or through gas/star accretion in the galaxy outskirts. The tight scaling relations found here strongly support mechanisms related to the dynamical response of discs to tidal forces rather than other scenarios. In fact, a gravitational-driven mechanism would have three advantages. The first is that this can be induced through a wide diversity of processes (such as those mentioned in Sect. 1). Secondly, it could provide a feasible explanation for the independence of these scaling relations of the Hubble type of the galaxy (although it must be confirmed more robustly, as explained above), since just a stellar disc and gravity are required to give rise to them. Finally, a gravitationally induced mechanism could also explain some of the apparently contradictory results with the environment discussed previously. If the processes triggering anti-truncations are mostly related to gravitational interactions, we expect to find them present in both groups and clusters and in similar fractions, because mergers and interactions can be equally relevant in both environments (Moran et al. 2007; Wilman et al. 2009; Plauchu-Frayn \& Coziol 2010a,b; Bekki \& Couch 2011; Vijayaraghavan \& Ricker 2013). Therefore, it is reasonable to find a weak dependence of their properties on the tidal interaction field (as reported by L14), but we do not expect to find significant trends of the break properties with the local density at the same time, because the anti-truncation can have formed through an interaction not related with the current environment of the galaxy.

In all cases, these speculative suggestions need to be confirmed through numerical simulations. At the moment, only Borlaff et al. (2014) have shown that major mergers are capable of producing anti-truncated S0 galaxies that obey these scaling relations using $N$-body simulations. Nevertheless, it is obvious that the role of major mergers in the formation of latetype spirals must have been quite limited, so at least the Type-III discs in Sbc-Sd galaxies require different mechanisms, which also have to predict these scaling relations. In particular, satellite accretions are known to produce anti-truncations (Laurikainen \& Salo 2001; Peñarrubia et al. 2006; Younger et al. 2007), inducing secular evolution in the disc that can couple the inner and outer galaxy structure at the same time (Eliche-Moral et al. 2006, 2011, 2012, 2013). This makes them good candidates to form anti-truncated stellar discs. However, additional studies demonstrating the feasibility of this and other mechanisms in reproducing the scaling relations found here are required.

\footnotetext{
2 The break strength of Type-II and Type-III profiles is defined as the logarithm of the outer-to-inner scalelengths ratio.
}

\section{Conclusions}

We have investigated whether the tight scaling relations recently observed by Borlaff et al. (2014) in Type-III S0s are satisfied by anti-truncated galaxies of other Hubble types. We have used the samples of Type-III galaxies published by E08 and G11 in the $R$ band and by L14 in Spitzer $3.6 \mu \mathrm{m}$ band, as well as the characterizations performed by these authors to the surface brightness profiles of these galaxies. The $R$-band dataset consists of 40 antitruncated galaxies with types spanning from $\mathrm{S} 0$ to $\mathrm{Sbc}$, while the $3.6 \mu \mathrm{m}$ sample has 62 galaxies of S0-Scd types. Nearly half of the galaxies in each sample are barred.

We have analysed the trends followed by S0s and spirals (all, Sa-Sab, Sb-Sbc, and Sc-Scd), as well as for barred and unbarred galaxies, in several planes relating the characteristic photometric parameters of the breaks $\left(\mu_{\text {brkIII }}, R_{\text {brkIII }}\right)$ and of the inner and outer discs of these anti-truncated galaxies $\left(\mu_{0, \mathrm{i}}, h_{\mathrm{i}}\right.$, $\mu_{0, \mathrm{o}}, h_{\mathrm{o}}$ ), for the $R$ and $3.6 \mu \mathrm{m}$ datasets separately. We have used the Spearman rank correlation test to select the correlations that are significant at the $95 \%$ confidence level. Linear fits have been performed to the trends followed by each galaxy type in each photometric plane, and the Pearson's coefficient has been used to measure the level of linear correlation.

We have obtained the following results:

1. The anti-truncated discs of spirals (taking them all together, or dividing them into Hubble classes) obey tight photometric relations, like those observed in $\mathrm{S} 0$ galaxies, both in the $R$ and $3.6 \mu \mathrm{m}$ bands.

2. The anti-truncated discs of barred and unbarred galaxies also follow tight photometric relations, again both in $R$ and $3.6 \mu \mathrm{m}$.

3. The majority of these correlations have high statistical significance despite the relatively low numbers of the available datasets, showing clear linear trends when $h_{\mathrm{i}}, h_{\mathrm{o}}, R_{\text {brkIII }}$, and $R_{25}$ are plotted on a logarithmic scale. This implies the existence of strong scaling relations in the Type-III discs of all Hubble types between their characteristic parameters $\left(h_{\mathrm{i}}, h_{\mathrm{o}}\right.$, $\left.\mu_{0, \mathrm{i}}, \mu_{0, \mathrm{o}}, \mu_{\mathrm{brkIII}}\right)$ and $R_{\text {brkIII }}$, as well as between the parameters of the inner and outer discs $\left(\mu_{0, \mathrm{i}}-h_{\mathrm{i}}, \mu_{0, \mathrm{o}}-h_{\mathrm{o}}\right.$, and $\left.h_{\mathrm{i}}-h_{\mathrm{o}}\right)$.

4. The correlations between $\mu_{0, i}, \mu_{0,0}$, or $\mu_{\text {brkIII }}$ with the logarithm of the characteristic scalelengths $\left(h_{\mathrm{i}}, h_{\mathrm{o}}\right.$, or $\left.R_{\text {brkIII }}\right)$ improve significantly when the scalelengths are normalized to $R_{25}$.

5. The logarithm of the characteristic scalelengths of antitruncated discs $\left(h_{\mathrm{i}}, h_{\mathrm{o}}\right.$, and $\left.R_{\text {brkIII }}\right)$ scale with $\log \left(R_{25}\right)$ for all galaxy types in $3.6 \mu \mathrm{m}$. In $R$, the linear trends are less tight and lose significance in spiral types.

6. The observational uncertainties of the data samples are too high to discern robustly whether the analysed scaling relations are similar in SOs and spirals, barred and unbarred galaxies, and in the $R$ and $3.6 \mu \mathrm{m}$ bands. However, no statistical evidence is found of significant differences between the relations followed by S0s and spirals and by barred and unbarred galaxies within the errors. This result suggests that the scaling relations of anti-truncated discs are independent of the morphological type and the presence or absence of bars. Deeper data and larger samples are required to confirm these results robustly.

In conclusion, the tight scaling relations found in the present study for Type-III discs impose strong constraints on any formation scenarios proposed to explain the formation of anti-truncations in stellar discs across the Hubble sequence, 
independently of whether the relations really depend on the morphological type or the hosting of a bar.

Acknowledgements. The authors thank the anonymous referee for the provided input that helped to improve this publication significantly. We acknowledge the usage of the HyperLeda database (http://leda.univ-lyon $1 . \mathrm{fr}$ ). This research has made use of the NASA Astrophysics Data System and NASA/IPAC Extragalactic Database (NED). Supported by the Ministerio de Economía y Competitividad del Gobierno de España (MINECO) under project AYA201231277, the Instituto de Astrofísica de Canarias under project P3/86, and the Consejo Nacional de Ciencia y Tecnología de México (CONACYT) under project 167236. J.E.B. acknowledges financial support to the DAGAL network from the People Programme (Marie Curie Actions) of the European Unions Seventh Framework Programme FP7/2007-2013/ under REA grant agreement number PITN-GA-2011-289313.

\section{References}

Bekki, K., \& Couch, W. J. 2011, MNRAS, 415, 1783

Blanton, M. R., \& Roweis, S. 2007, AJ, 133, 734

Borlaff, A., Eliche-Moral, M. C., Rodríguez-Pérez, C., et al. 2014, A\&A, 570, A103

Buta, R., Sheth, K., Athanassoula, E., et al. 2015, ApJS, 217, 32

Comerón, S., Elmegreen, B. G., Salo, H., et al. 2012, ApJ, 759, 98

de Vaucouleurs, G. 1957, AJ, 62, 69

de Vaucouleurs, G. 1958, ApJ, 128, 465

de Vaucouleurs, G., de Vaucouleurs, A., Corwin, Jr., H. G., et al. 1991, Third Reference Catalogue of Bright Galaxies, Vol. I: Explanations and references, Vol. II: Data for galaxies between $0 \mathrm{~h}$ and $12 \mathrm{~h}$, Vol. III: Data for galaxies between $12 \mathrm{~h}$ and $24 \mathrm{~h}$ (New York, USA: Springer)

Eliche-Moral, M. C., Balcells, M., Aguerri, J. A. L., \& González-García, A. C. 2006, A\&A, 457, 91

Eliche-Moral, M. C., González-García, A. C., Balcells, M., et al. 2011, A\&A, 533, A104

Eliche-Moral, M. C., González-García, A. C., Aguerri, J. A. L., et al. 2012, A\&A, 547, A48

Eliche-Moral, M. C., González-García, A. C., Aguerri, J. A. L., et al. 2013, A\&A, 552, A67

Elmegreen, B. G., \& Hunter, D. A. 2006, ApJ, 636, 712
Erwin, P., Beckman, J. E., \& Pohlen, M. 2005, ApJ, 626, L81 Erwin, P., Pohlen, M., \& Beckman, J. E. 2008, AJ, 135, 20 (E08) Erwin, P., Gutiérrez, L., \& Beckman, J. E. 2012, ApJ, 744, L11

Freeman, K. C. 1970, ApJ, 160, 811

Gregg, M. D. 1989, ApJS, 69, 217

Gutiérrez, L., Erwin, P., Aladro, R., \& Beckman, J. E. 2011, AJ, 142, 145 (G11)

Herpich, J., Stinson, G. S., Dutton, A. A., et al. 2015, MNRAS, 448, L99

Ilyina, M. A., \& Sil'chenko, O. K. 2012, Astron. Astrophys. Trans., 27, 313

Kazantzidis, S., Zentner, A. R., Kravtsov, A. V., Bullock, J. S., \& Debattista, V. P. 2009, ApJ, 700, 1896

Kim, T., Gadotti, D. A., Sheth, K., et al. 2014, ApJ, 782, 64

Laine, J., Laurikainen, E., Salo, H., et al. 2014, MNRAS, 441, 1992 (L14)

Lang, M., Holley-Bockelmann, K., \& Sinha, M. 2014, ApJ, 790, L33

Laurikainen, E., \& Salo, H. 2001, MNRAS, 324, 685

Laurikainen, E., Salo, H., Buta, R., \& Knapen, J. H. 2011, MNRAS, 418, 1452

Maltby, D. T., Aragón-Salamanca, A., Gray, M. E., et al. 2015, MNRAS, 447, 1506

Maltby, D. T., Gray, M. E., Aragón-Salamanca, A., et al. 2012a, MNRAS, 419, 669

Maltby, D. T., Hoyos, C., Gray, M. E., Aragón-Salamanca, A., \& Wolf, C. 2012b, MNRAS, 420, 2475

Minchev, I., Famaey, B., Quillen, A. C., et al. 2012, A\&A, 548, A126

Möllenhoff, C. 2004, A\&A, 415, 63

Moran, S. M., Ellis, R. S., Treu, T., et al. 2007, ApJ, 671, 1503

Patterson, F. S. 1940, Harvard College Observatory Bulletin, 914, 9

Peñarrubia, J., McConnachie, A., \& Babul, A. 2006, ApJ, 650, L33

Plauchu-Frayn, I., \& Coziol, R. 2010a, AJ, 139, 2643

Plauchu-Frayn, I., \& Coziol, R. 2010b, AJ, 140, 612

Roediger, J. C., Courteau, S., Sánchez-Blázquez, P., \& McDonald, M. 2012, ApJ, 758,41

Sheth, K., Regan, M., Hinz, J. L., et al. 2010, PASP, 122, 1397

Sil'chenko, O. K. 2009, in IAU Symp. 254, eds. J. Andersen, Nordströara, B. M, \& J. Bland-Hawthorn, 173

van der Kruit, P. C. 1979, A\&AS, 38, 15

van der Kruit, P. C. 1987, A\&A, 173, 59

Vijayaraghavan, R., \& Ricker, P. M. 2013, MNRAS, 435, 2713

Walker, I. R., Mihos, J. C., \& Hernquist, L. 1996, ApJ, 460, 121

Wilman, D. J., Oemler, Jr., A., Mulchaey, J. S., et al. 2009, ApJ, 692, 298

Younger, J. D., Cox, T. J., Seth, A. C., \& Hernquist, L. 2007, ApJ, 670, 269

Younger, J. D., Besla, G., Cox, T. J., et al. 2008, ApJ, 676, L21 
M. C. Eliche-Moral et al.: Scaling relations of anti-truncated stellar discs in S0-Scd galaxies

Table 2. Linear fits performed to the trends of Type-III galaxies in the photometric planes relating $h_{\mathrm{i}}$ and $h_{\mathrm{o}}$ with $R_{\text {brkIII. }}$.

\begin{tabular}{|c|c|c|c|c|c|c|c|c|}
\hline \multicolumn{9}{|c|}{ 1) $\log \left(h_{\mathrm{i}}\right)$ vs. $\log \left(R_{\mathrm{brk}}\right)$} \\
\hline & All & Spirals & S0 & $\mathrm{Sa}-\mathrm{Sab}$ & $\mathrm{Sb}-\mathrm{Sbc}$ & Sc-Scd & Barred & Unbarred \\
\hline \multirow[t]{2}{*}{$m$} & $0.71_{-0.16}^{+0.17}$ & $0.65_{-0.34}^{+0.28}$ & $0.80_{-0.25}^{+0.34}$ & $0.38_{-0.29}^{+0.35}$ & $0.90_{-0.41}^{+0.58}$ & $\ldots$ & $0.82_{-0.15}^{+0.26}$ & $0.69_{-0.26}^{+0.31}$ \\
\hline & $1.006_{-0.087}^{+0.089}$ & $0.97_{-0.11}^{+0.15}$ & $1.053_{-0.100}^{+0.095}$ & $0.98_{-0.23}^{+0.24}$ & $0.91_{-0.36}^{+0.23}$ & $0.85_{-0.26}^{+0.35}$ & $0.96_{-0.15}^{+0.15}$ & $1.05_{-0.15}^{+0.14}$ \\
\hline \multirow[t]{2}{*}{$C_{0}$} & $-0.36_{-0.16}^{+0.15}$ & $-0.30_{-0.25}^{+0.34}$ & $-0.44_{-0.32}^{+0.24}$ & $-0.02_{-0.35}^{+0.30}$ & $-0.55_{-0.54}^{+0.33}$ & $\ldots$ & $-0.48_{-0.23}^{+0.14}$ & $-0.32_{-0.29}^{+0.25}$ \\
\hline & $-0.613_{-0.081}^{+0.081}$ & $-0.62_{-0.13}^{+0.11}$ & $-0.59_{-0.10}^{+0.10}$ & $-0.62_{-0.22}^{+0.26}$ & $-0.57_{-0.17}^{+0.36}$ & $-0.57_{-0.25}^{+0.27}$ & $-0.59_{-0.14}^{+0.14}$ & $-0.62_{-0.13}^{+0.14}$ \\
\hline \multirow[t]{2}{*}{$\rho$} & 0.823 & 0.740 & 0.877 & 0.706 & 0.857 & 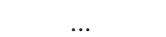 & 0.856 & 0.761 \\
\hline & 0.896 & 0.869 & 0.939 & 0.791 & 0.775 & 0.833 & 0.932 & 0.861 \\
\hline \multirow[t]{2}{*}{$p_{S}$} & $7.08 \mathrm{e}-11$ & $2.89 \mathrm{e}-04$ & $1.87 \mathrm{e}-07$ & $1.02 \mathrm{e}-02$ & $1.37 \mathrm{e}-02$ & & $2.34 \mathrm{e}-05$ & $1.55 \mathrm{e}-05$ \\
\hline & $7.96 e-23$ & $2.39 \mathrm{e}-10$ & $5.24 \mathrm{e}-15$ & $2.62 \mathrm{e}-04$ & $4.08 \mathrm{e}-02$ & $1.02 \mathrm{e}-02$ & $1.90 \mathrm{e}-13$ & $1.35 \mathrm{e}-10$ \\
\hline \multirow[t]{4}{*}{$N_{\text {pairs }}$} & 40 & 19 & 21 & 12 & 7 & $\ldots$ & 16 & 24 \\
\hline & 62 & 31 & 31 & 16 & 7 & 8 & 29 & 33 \\
\hline & \multicolumn{8}{|c|}{ 2) $\log \left(h_{\mathrm{i}} / R_{25}\right)$ vs. $\log \left(R_{\mathrm{brkIII}} / R_{25}\right)$} \\
\hline & All & Spirals & So & $\mathrm{Sa}-\mathrm{Sab}$ & $\mathrm{Sb}-\mathrm{Sbc}$ & Sc-Scd & Barred & Unbarred \\
\hline \multirow[t]{2}{*}{$m$} & $0.62_{-0.13}^{+0.14}$ & $0.60_{-0.22}^{+0.17}$ & $0.65_{-0.26}^{+0.52}$ & $0.52_{-0.28}^{+0.23}$ & $0.77_{-0.74}^{+0.52}$ & $\ldots$ & $0.77_{-0.16}^{+0.21}$ & $0.57_{-0.20}^{+0.28}$ \\
\hline & $0.649_{-0.099}^{+0.113}$ & $0.65_{-0.20}^{+0.27}$ & $0.74_{-0.12}^{+0.12}$ & $0.56_{-0.19}^{+0.36}$ & $0.88_{-0.37}^{+0.23}$ & $0.85_{-1.21}^{+0.52}$ & $0.67_{-0.12}^{+0.19}$ & $0.67_{-0.18}^{+0.19}$ \\
\hline \multirow[t]{2}{*}{$C_{0}$} & $-0.619_{-0.023}^{+0.024}$ & $-0.629_{-0.038}^{+0.037}$ & $-0.608_{-0.054}^{+0.046}$ & $-0.627_{-0.049}^{+0.042}$ & $-0.641_{-0.081}^{+0.060}$ & $\ldots$ & $-0.636_{-0.038}^{+0.039}$ & $-0.601_{-0.040}^{+0.042}$ \\
\hline & $-0.625_{-0.018}^{+0.018}$ & $-0.657_{-0.026}^{+0.026}$ & $-0.569_{-0.031}^{+0.030}$ & $-0.644_{-0.038}^{+0.040}$ & $-0.651_{-0.037}^{+0.037}$ & $-0.686_{-0.047}^{+0.042}$ & $-0.637_{-0.027}^{+0.026}$ & $-0.597_{-0.037}^{+0.035}$ \\
\hline \multirow[t]{2}{*}{$\rho$} & 0.800 & 0.721 & 0.806 & 0.762 & 0.750 & & 0.847 & 0.766 \\
\hline & 0.801 & 0.860 & 0.871 & 0.782 & 0.893 & 0.619 & 0.859 & 0.770 \\
\hline \multirow[t]{2}{*}{$p_{S}$} & $5.79 \mathrm{e}-10$ & $4.95 \mathrm{e}-04$ & $1.01 \mathrm{e}-05$ & $3.95 \mathrm{e}-03$ & $5.22 \mathrm{e}-02$ & $\ldots$ & $3.47 \mathrm{e}-05$ & $1.28 \mathrm{e}-05$ \\
\hline & $5.42 \mathrm{e}-15$ & $5.86 \mathrm{e}-10$ & $1.87 \mathrm{e}-10$ & $3.41 \mathrm{e}-04$ & $6.81 \mathrm{e}-03$ & $1.02 \mathrm{e}-01$ & $2.45 \mathrm{e}-09$ & $1.64 \mathrm{e}-07$ \\
\hline \multirow{4}{*}{$N_{\text {pairs }}$} & 40 & 19 & 21 & 12 & 7 & $\ldots$ & 16 & 24 \\
\hline & 62 & 31 & 31 & 16 & 7 & 8 & 29 & 33 \\
\hline & & & & $\log \left(h_{\mathrm{o}}\right)$ vs. $\log$ & $\left.R_{\text {brkIII }}\right)$ & & & \\
\hline & All & Spirals & S0 & $\mathrm{Sa}-\mathrm{Sab}$ & $\mathrm{Sb}-\mathrm{Sbc}$ & Sc-Scd & Barred & Unbarred \\
\hline \multirow[t]{2}{*}{$m$} & $0.72_{-0.24}^{+0.24}$ & $0.49_{-0.47}^{+0.35}$ & $1.00_{-0.40}^{+0.44}$ & $0.31_{-0.31}^{+0.40}$ & $0.51_{-2.28}^{+0.70}$ & $\ldots$ & $0.27_{-0.45}^{+0.28}$ & $0.99_{-0.37}^{+0.36}$ \\
\hline & $1.08_{-0.12}^{+0.14}$ & $1.03_{-0.18}^{+0.30}$ & $1.16_{-0.19}^{+0.18}$ & $1.05_{-0.29}^{+0.45}$ & $0.67_{-0.44}^{+0.66}$ & $0.98_{-0.29}^{+0.42}$ & $1.05_{-0.26}^{+0.43}$ & $1.12_{-0.17}^{+0.24}$ \\
\hline \multirow[t]{2}{*}{$C_{0}$} & $0.04_{-0.21}^{+0.23}$ & $0.26_{-032}^{+0.45}$ & $-0.25_{-0.42}^{+0.34}$ & $0.47_{-0.37}^{+0.30}$ & $0.16_{-047}^{+2.17}$ & $\ldots$ & $0.39_{-021}^{+0.43}$ & $-0.20_{-0.34}^{+0.36}$ \\
\hline & $-0.39_{-0.12}^{+0.11}$ & $-0.38_{-0.24}^{+0.17}$ & $-0.43_{-0.16}^{+0.19}$ & $-0.37_{-0.37}^{+0.33}$ & $-0.07_{-0.52}^{+0.43}$ & $-0.44_{-0.35}^{+0.31}$ & $-0.42_{-0.22}^{+0.25}$ & $-0.39_{-0.20}^{+0.16}$ \\
\hline \multirow[t]{2}{*}{$\rho$} & 0.594 & 0.391 & 0.798 & 0.448 & 0.107 & $\ldots$ & 0.363 & 0.675 \\
\hline & 0.868 & 0.839 & 0.900 & 0.824 & 0.714 & 0.976 & 0.918 & 0.882 \\
\hline \multirow[t]{2}{*}{$p_{S}$} & $1.07 \mathrm{e}-04$ & $9.77 \mathrm{e}-02$ & $7.30 \mathrm{e}-05$ & $1.45 \mathrm{e}-01$ & $8.19 \mathrm{e}-01$ & $\ldots$ & $2.23 \mathrm{e}-01$ & $2.98 \mathrm{e}-04$ \\
\hline & $7.07 e-20$ & $3.83 \mathrm{e}-09$ & $5.97 \mathrm{e}-12$ & $8.84 \mathrm{e}-05$ & $7.13 \mathrm{e}-02$ & $3.31 \mathrm{e}-05$ & $2.45 \mathrm{e}-12$ & $1.14 \mathrm{e}-11$ \\
\hline \multirow[t]{4}{*}{$N_{\text {pairs }}$} & 37 & 19 & 18 & 12 & 7 & $\ldots$ & 13 & 24 \\
\hline & 62 & 31 & 31 & 16 & 7 & 8 & 29 & 33 \\
\hline & & & 4) $\log$ & $\left(h_{\mathrm{o}} / R_{25}\right)$ vs. $\log$ & $\left.R_{\text {brkIII }} / R_{25}\right)$ & & & \\
\hline & All & Spirals & So & $\mathrm{Sa}-\mathrm{Sab}$ & $\mathrm{Sb}-\mathrm{Sbc}$ & Sc-Scd & Barred & Unbarred \\
\hline \multirow[t]{2}{*}{$m$} & $0.60_{-0.17}^{+0.18}$ & $0.54_{-0.31}^{+0.30}$ & $0.80_{-0.39}^{+0.60}$ & $0.42_{-0.23}^{+0.24}$ & $0.8_{-2.5}^{+1.1}$ & $\ldots$ & $0.31_{-0.61}^{+0.29}$ & $0.76_{-0.26}^{+0.38}$ \\
\hline & $0.73_{-0.18}^{+0.22}$ & $0.59_{-0.25}^{+0.42}$ & $0.92_{-0.32}^{+0.37}$ & $0.49_{-0.27}^{+0.55}$ & $0.81_{-0.70}^{+0.48}$ & $1.2_{-1.0}^{+1.2}$ & $0.85_{-0.34}^{+0.64}$ & $0.75_{-0.28}^{+0.32}$ \\
\hline \multirow[t]{2}{*}{$C_{0}$} & $-0.220_{-0.040}^{+0.041}$ & $-0.216_{-0.068}^{+0.066}$ & $-0.245_{-0.082}^{+0.080}$ & $-0.206_{-0.058}^{+0.056}$ & $-0.25_{-0.17}^{+0.14}$ & $\ldots$ & $-0.259_{-0.076}^{+0.072}$ & $-0.208_{-0.066}^{+0.066}$ \\
\hline & $-0.333_{-0.030}^{+0.033}$ & $-0.363_{-0.043}^{+0.052}$ & $-0.298_{-0.060}^{+0.058}$ & $-0.324_{-0.066}^{+0.086}$ & $-0.353_{-0.093}^{+0.089}$ & $-0.441_{-0.047}^{+0.073}$ & $-0.381_{-0.044}^{+0.055}$ & $-0.293_{-0.052}^{+0.057}$ \\
\hline \multirow[t]{2}{*}{$\rho$} & 0.614 & 0.619 & 0.581 & 0.608 & 0.571 & $\ldots$ & 0.264 & 0.753 \\
\hline & 0.753 & 0.683 & 0.865 & 0.624 & 0.643 & 0.786 & 0.893 & 0.739 \\
\hline \multirow[t]{2}{*}{$p_{S}$} & $5.30 \mathrm{e}-05$ & $4.69 e-03$ & $1.15 \mathrm{e}-02$ & $3.58 \mathrm{e}-02$ & $1.80 \mathrm{e}-01$ & $\ldots$ & $3.84 \mathrm{e}-01$ & $2.17 \mathrm{e}-05$ \\
\hline & $1.65 \mathrm{e}-12$ & $2.29 \mathrm{e}-05$ & $3.36 \mathrm{e}-10$ & $9.86 \mathrm{e}-03$ & $1.19 \mathrm{e}-01$ & $2.08 \mathrm{e}-02$ & $7.69 \mathrm{e}-11$ & $9.21 \mathrm{e}-07$ \\
\hline$N_{\text {pairs }}$ & 37 & 19 & 18 & 12 & 7 & $\ldots$ & 13 & 24 \\
\hline & 62 & 31 & 31 & 16 & 7 & 8 & 29 & 33 \\
\hline
\end{tabular}

Notes. For each photometric relation, we list the results obtained from the linear fits performed to the various galaxy subsamples in columns (all galaxies, spirals, S0s, Sa-Sab's, Sb-Sbc's, Sc-Scd's, barred galaxies, and unbarred galaxies). The first row of results in each parameter (for each relation and galaxy subsample considered) corresponds to the fits performed to the data in the $R$ band, while the second row corresponds to the results in the $3.6 \mu \mathrm{m}$ band. In each linear fit, we provide the slope $(m)$, the $Y$-intercept value $\left(C_{0}\right)$, the Pearson coefficient of linear correlation $(\rho)$, the Spearman rank probability of random correlation $\left(p_{\mathrm{S}}\right)$, and the number of data pairs available for each fit (see Sect. 3 for more details). 
A\&A 580, A33 (2015)

Table 3. Linear fits performed to the trends of Type-III galaxies in the planes relating $\mu_{0, \mathrm{i}}$ and $\mu_{0, \mathrm{o}}$ with $R_{\mathrm{brkIII}}$.

\begin{tabular}{|c|c|c|c|c|c|c|c|c|}
\hline \multicolumn{9}{|c|}{ 5) $\mu_{0, \mathrm{i}}$ vs. $\log$} \\
\hline & All & Spirals & So & $\mathrm{Sa}-\mathrm{Sab}$ & $\mathrm{Sb}-\mathrm{Sbc}$ & Sc-Scd & Barred & Unbarred \\
\hline \multirow[t]{2}{*}{$m$} & $3.1_{-1.3}^{+1.7}$ & $2.9_{-2.7}^{+2.4}$ & $3.5_{-1.9}^{+3.2}$ & $1.5_{-3.1}^{+2.7}$ & $4.5_{-7.6}^{+3.4}$ & $\ldots$ & $4.8_{-2.2}^{+2.3}$ & $2.5_{-1.8}^{+2.8}$ \\
\hline & $1.72_{-0.91}^{+0.91}$ & $1.0_{-1.3}^{+1.5}$ & $2.6_{-1.1}^{+1.1}$ & $1.1_{-2.3}^{+1.9}$ & $0.4_{-5.5}^{+4.7}$ & $-0.9_{-2.3}^{+4.5}$ & $2.0_{-1.6}^{+1.3}$ & $1.9_{-1.5}^{+1.4}$ \\
\hline \multirow[t]{2}{*}{$C_{0}$} & $16.5_{-1.4}^{+1.2}$ & $16.5_{-2.2}^{+2.6}$ & $16.4_{-2.7}^{+1.5}$ & $17.9_{-2.5}^{+2.7}$ & $15.1_{-2.7}^{+7.4}$ & $\ldots$ & $15.0_{-2.0}^{+2.1}$ & $17.2_{-2.3}^{+1.4}$ \\
\hline & $17.43_{-0.77}^{+0.79}$ & $17.9_{-1.2}^{+1.2}$ & $16.9_{-1.0}^{+1.1}$ & $18.0_{-1.6}^{+2.4}$ & $18.1_{-3.8}^{+5.5}$ & $19.2_{-3.0}^{+2.4}$ & $17.3_{-1.1}^{+1.5}$ & $17.3_{-1.2}^{+1.2}$ \\
\hline \multirow{2}{*}{$\rho$} & 0.595 & 0.484 & 0.703 & 0.436 & 0.643 & & 0.829 & 0.447 \\
\hline & 0.508 & 0.292 & 0.687 & 0.394 & -0.214 & -0.310 & 0.499 & 0.439 \\
\hline \multirow{2}{*}{$p_{S}$} & $6.46 \mathrm{e}-05$ & $4.18 \mathrm{e}-02$ & $3.78 \mathrm{e}-04$ & $1.80 \mathrm{e}-01$ & $1.19 \mathrm{e}-01$ & & $1.32 \mathrm{e}-04$ & $2.85 \mathrm{e}-02$ \\
\hline & $2.51 \mathrm{e}-05$ & $1.11 \mathrm{e}-01$ & $1.96 \mathrm{e}-05$ & $1.31 \mathrm{e}-01$ & $6.45 \mathrm{e}-01$ & $4.56 \mathrm{e}-01$ & $5.82 \mathrm{e}-03$ & $1.05 \mathrm{e}-02$ \\
\hline \multirow[t]{4}{*}{$N_{\text {pairs }}$} & 39 & 18 & 21 & 11 & 7 & $\ldots$ & 15 & 24 \\
\hline & 62 & 31 & 31 & 16 & 7 & 8 & 29 & 33 \\
\hline & & & & ,i Vs. Iog & II $\left./ R_{25}\right)$ & & & \\
\hline & All & Spirals & S0 & $\mathrm{Sa}-\mathrm{Sab}$ & $\mathrm{Sb}-\mathrm{Sbc}$ & Sc-Scd & Barred & Unbarred \\
\hline \multirow[t]{2}{*}{$m$} & $3.3_{-13}^{+1.7}$ & $2.9_{-19}^{+1.9}$ & $4.4_{-28}^{+6.3}$ & $1.7_{-20}^{+2.0}$ & $5.3_{-24}^{+2.7}$ & $\ldots$ & $5.3_{-22}^{+1.1}$ & $2.9_{-18}^{+3.3}$ \\
\hline & $2.0_{-1.3}^{+1.2}$ & $0.7_{-2.4}^{+2.6}$ & $4.0_{-1.3}^{+1.2}$ & $0.8_{-3.3}^{+2.7}$ & $-0.9_{-2.5}^{+7.1}$ & $3.8_{-10.7}^{+7.4}$ & $3.5_{-1.3}^{+1.3}$ & $1.6_{-2.4}^{+2.4}$ \\
\hline \multirow[t]{2}{*}{$C_{0}$} & $19.29_{-0.21}^{+0.23}$ & $19.24_{-0.35}^{+0.31}$ & $19.28_{-0.50}^{+0.48}$ & $19.42_{-0.46}^{+0.36}$ & $18.92_{-0.45}^{+0.39}$ & $\ldots$ & $19.13_{-0.31}^{+0.33}$ & $19.40_{-0.35}^{+0.44}$ \\
\hline & $19.09_{-0.21}^{+0.21}$ & $18.84_{-0.30}^{+0.30}$ & $19.58_{-0.29}^{+0.25}$ & $19.14_{-0.38}^{+0.37}$ & $18.37_{-0.61}^{-0.45}$ & $18.54_{-0.59}^{+0.56}$ & $19.20_{-0.28}^{+0.26}$ & $19.11_{-0.43}^{+0.35}$ \\
\hline \multirow[t]{2}{*}{$\rho$} & 0.702 & 0.616 & 0.788 & 0.627 & 0.750 & & 0.781 & 0.617 \\
\hline & 0.431 & 0.194 & 0.747 & 0.113 & -0.143 & 0.095 & 0.611 & 0.268 \\
\hline \multirow[t]{2}{*}{$p_{S}$} & $6.52 \mathrm{e}-07$ & $6.48 \mathrm{e}-03$ & $2.20 \mathrm{e}-05$ & $3.88 \mathrm{e}-02$ & $5.22 \mathrm{e}-02$ & & $5.87 \mathrm{e}-04$ & $1.33 \mathrm{e}-03$ \\
\hline & $4.70 \mathrm{e}-04$ & $2.95 \mathrm{e}-01$ & $1.37 \mathrm{e}-06$ & $6.76 \mathrm{e}-01$ & $7.60 \mathrm{e}-01$ & $8.23 \mathrm{e}-01$ & $4.31 \mathrm{e}-04$ & $1.32 \mathrm{e}-01$ \\
\hline \multirow{4}{*}{$N_{\text {pairs }}$} & 39 & 18 & 21 & 11 & 7 & $\ldots$ & 15 & 24 \\
\hline & 62 & 31 & 31 & 16 & 7 & 8 & 29 & 33 \\
\hline & & & & $l_{0, \mathrm{o}} \mathrm{VS}$ & brkIII） & & & \\
\hline & All & Spirals & So & $\mathrm{Sa}-\mathrm{Sab}$ & $\mathrm{Sb}-\mathrm{Sbc}$ & Sc-Scd & Barred & Unbarred \\
\hline \multirow[t]{2}{*}{$m$} & $4.8_{-1.4}^{+1.3}$ & $4.7_{-2.9}^{+2.3}$ & $5.0_{-2.6}^{+1.9}$ & $6.2_{-1.9}^{+2.2}$ & $3.2_{-20.0}^{+6.8}$ & $\ldots$ & $3.2_{-4.7}^{+3.7}$ & $5.4_{-1.8}^{+1.7}$ \\
\hline & $2.06_{-0.94}^{+1.10}$ & $1.5_{-1.3}^{+1.7}$ & $2.8_{-1.7}^{+1.9}$ & $1.5_{-2.0}^{+2.6}$ & $-0.5_{-6.3}^{+5.5}$ & $1.2_{-3.0}^{+2.6}$ & $2.7_{-1.7}^{+1.7}$ & $1.9_{-1.4}^{+2.0}$ \\
\hline \multirow[t]{2}{*}{$C_{0}$} & $17.6_{-1.3}^{+1.3}$ & $17.7_{-2.3}^{+2.8}$ & $17.5_{-1.8}^{+2.1}$ & $16.1_{-2.2}^{+1.8}$ & $18.8_{-4.6}^{+19.2}$ & $\ldots$ & $18.9_{-3.5}^{+4.6}$ & $17.2_{-1.7}^{+1.5}$ \\
\hline & $19.25_{-0.94}^{+0.85}$ & $19.8_{-1.4}^{+1.2}$ & $18.4_{-1.6}^{+1.5}$ & $20.0_{-2.2}^{+2.1}$ & $21.1_{-4.6}^{+6.1}$ & $19.9_{-2.0}^{+2.3}$ & $18.7_{-1.5}^{+1.6}$ & $19.2_{-1.7}^{+1.3}$ \\
\hline \multirow[t]{2}{*}{$\rho$} & 0.644 & 0.561 & 0.712 & 0.827 & 0.250 & & 0.467 & 0.703 \\
\hline & 0.452 & 0.339 & 0.569 & 0.409 & 0.071 & 0.405 & 0.465 & 0.416 \\
\hline \multirow[t]{2}{*}{$p_{S}$} & $3.93 \mathrm{e}-05$ & $1.55 \mathrm{e}-02$ & $1.98 \mathrm{e}-03$ & $1.68 \mathrm{e}-03$ & $5.89 \mathrm{e}-01$ & & $1.74 \mathrm{e}-01$ & $1.29 \mathrm{e}-04$ \\
\hline & $2.28 \mathrm{e}-04$ & $6.23 \mathrm{e}-02$ & $8.47 \mathrm{e}-04$ & $1.16 \mathrm{e}-01$ & $8.79 \mathrm{e}-01$ & $3.20 \mathrm{e}-01$ & $1.10 \mathrm{e}-02$ & $1.60 \mathrm{e}-02$ \\
\hline \multirow[t]{4}{*}{$N_{\text {pairs }}$} & 34 & 18 & 16 & 11 & 7 & $\ldots$ & 10 & 24 \\
\hline & 62 & 31 & 31 & 16 & 7 & 8 & 29 & 33 \\
\hline & & & & vs. $\log (I$ & III $\left./ R_{25}\right)$ & & & \\
\hline & All & Spirals & S0 & $\mathrm{Sa}-\mathrm{Sab}$ & $\mathrm{Sb}-\mathrm{Sbc}$ & Sc-Scd & Barred & Unbarred \\
\hline \multirow[t]{2}{*}{$m$} & $5.5_{-1.1}^{+1.2}$ & $5.4_{-1.7}^{+1.6}$ & $5.9_{-2.8}^{+4.9}$ & $4.9_{-1.4}^{+2.5}$ & $6.5_{-10.3}^{+3.6}$ & $\ldots$ & $4.6_{-5.8}^{+2.1}$ & $5.8_{-1.7}^{+2.7}$ \\
\hline & $4.0_{-1.5}^{+1.4}$ & $2.5_{-3.0}^{+2.8}$ & $5.9_{-1.7}^{+2.0}$ & $2.9_{-4.5}^{+2.9}$ & $-0.4_{-5.4}^{+8.1}$ & $7.6_{-7.2}^{+4.4}$ & $6.0_{-1.7}^{+2.8}$ & $3.5_{-2.5}^{+2.3}$ \\
\hline \multirow[t]{2}{*}{$C_{0}$} & $21.97_{-0.25}^{+0.26}$ & $22.01_{-0.36}^{+0.40}$ & $21.81_{-0.61}^{+0.55}$ & $22.23_{-0.35}^{+0.40}$ & $21.63_{-0.66}^{+0.99}$ & $\ldots$ & $21.77_{-0.55}^{+0.69}$ & $22.02_{-0.42}^{+0.41}$ \\
\hline & $21.31_{-0.22}^{+0.23}$ & $21.24_{-0.34}^{+0.37}$ & $21.42_{-0.32}^{+0.31}$ & $21.59_{-0.45}^{+0.56}$ & $20.74_{-0.74}^{+0.74}$ & $20.86_{-0.43}^{+0.40}$ & $21.29_{-0.27}^{+0.30}$ & $21.27_{-0.40}^{+0.42}$ \\
\hline \multirow[t]{2}{*}{$\rho$} & 0.774 & 0.819 & 0.709 & 0.836 & 0.786 & & 0.612 & 0.834 \\
\hline & 0.667 & 0.435 & 0.844 & 0.409 & -0.143 & 0.019 & 0.859 & 0.488 \\
\hline \multirow[t]{2}{*}{$p_{S}$} & $7.76 \mathrm{e}-08$ & $3.25 \mathrm{e}-05$ & $2.11 \mathrm{e}-03$ & $1.33 \mathrm{e}-03$ & $3.62 \mathrm{e}-02$ & & $6.00 \mathrm{e}-02$ & $4.15 \mathrm{e}-07$ \\
\hline & $3.13 \mathrm{e}-09$ & $1.44 \mathrm{e}-02$ & $2.38 \mathrm{e}-09$ & $1.16 \mathrm{e}-01$ & $7.60 \mathrm{e}-01$ & $1.02 \mathrm{e}-01$ & $2.45 \mathrm{e}-09$ & $4.00 \mathrm{e}-03$ \\
\hline \multirow[t]{2}{*}{$N_{\text {pairs }}$} & 34 & 18 & 16 & 11 & 7 & $\ldots$ & 10 & 24 \\
\hline & 62 & 31 & 31 & 16 & 7 & 8 & 29 & 33 \\
\hline
\end{tabular}

Notes. See the notes of Table 2. 
M. C. Eliche-Moral et al.: Scaling relations of anti-truncated stellar discs in S0-Scd galaxies

Table 4. Linear fits performed to the trends of Type-III galaxies in the photometric planes relating $\mu_{\text {brkIII }}$ with $R_{\text {brkIIII }}$.

\begin{tabular}{|c|c|c|c|c|c|c|c|c|}
\hline \multicolumn{9}{|c|}{ 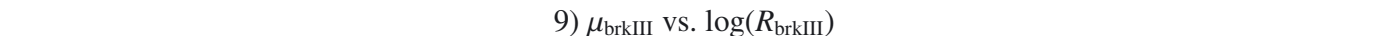 } \\
\hline & All & Spirals & S0 & $\mathrm{Sa}-\mathrm{Sab}$ & $\mathrm{Sb}-\mathrm{Sbc}$ & Sc-Scd & Barred & Unbarred \\
\hline \multirow[t]{2}{*}{$m$} & $6.0_{-1.5}^{+1.5}$ & $7.2_{-3.3}^{+2.6}$ & $4.6_{-2.5}^{+1.7}$ & $9.3_{-2.1}^{+2.2}$ & $6.0_{-9.1}^{+5.9}$ & $\ldots$ & $7.0_{-2.6}^{+3.1}$ & $5.1_{-2.3}^{+2.2}$ \\
\hline & $1.76_{-0.77}^{+0.80}$ & $1.5_{-1.1}^{+1.0}$ & $2.0_{-1.5}^{+1.6}$ & $1.4_{-1.5}^{+1.6}$ & $1.5_{-4.4}^{+2.9}$ & $1.2_{-3.8}^{+2.3}$ & $2.4_{-1.7}^{+1.6}$ & $1.5_{-1.1}^{+1.2}$ \\
\hline \multirow[t]{2}{*}{$C_{0}$} & $18.2_{-1.5}^{+1.4}$ & $17.0_{-2.6}^{+3.1}$ & $19.7_{-1.4}^{+2.1}$ & $14.7_{-2.5}^{+2.1}$ & $18.5_{-4.8}^{+8.5}$ & ... & $17.7_{-3.1}^{+2.4}$ & $19.0_{-2.3}^{+2.0}$ \\
\hline & $21.83_{-0.79}^{+0.76}$ & $22.3_{-1.0}^{+1.1}$ & $21.2_{-1.6}^{+1.5}$ & $22.4_{-1.8}^{+1.7}$ & $21.8_{-2.3}^{+4.5}$ & $22.9_{-1.9}^{+2.9}$ & $21.6_{-1.7}^{+1.7}$ & $21.7_{-1.2}^{+1.1}$ \\
\hline \multirow[t]{2}{*}{$\rho$} & 0.712 & 0.773 & 0.703 & 0.897 & 0.643 & $\ldots$ & 0.964 & 0.623 \\
\hline & 0.380 & 0.397 & 0.411 & 0.364 & 0.559 & 0.310 & 0.385 & 0.345 \\
\hline \multirow[t]{2}{*}{$p_{S}$} & $2.39 \mathrm{e}-06$ & $1.70 \mathrm{e}-04$ & $2.38 \mathrm{e}-03$ & $1.78 \mathrm{e}-04$ & $1.19 \mathrm{e}-01$ & $\ldots$ & $7.32 \mathrm{e}-06$ & $1.15 \mathrm{e}-03$ \\
\hline & $2.29 \mathrm{e}-03$ & $2.68 \mathrm{e}-02$ & $2.16 \mathrm{e}-02$ & $1.66 \mathrm{e}-01$ & $1.92 \mathrm{e}-01$ & $4.56 \mathrm{e}-01$ & $3.91 \mathrm{e}-02$ & $4.93 \mathrm{e}-02$ \\
\hline \multirow{4}{*}{$N_{\text {pairs }}$} & 34 & 18 & 16 & 11 & 7 & ... & 10 & 24 \\
\hline & 62 & 31 & 31 & 16 & 7 & 8 & 29 & 33 \\
\hline & & & 10) & rkIII Vs. $\log ($ & brkIII $\left./ R_{25}\right)$ & & & \\
\hline & All & Spirals & So & $\mathrm{Sa}-\mathrm{Sab}$ & $\mathrm{Sb}-\mathrm{Sbc}$ & Sc-Scd & Barred & Unbarred \\
\hline \multirow[t]{2}{*}{$m$} & $7.38_{-0.74}^{+0.80}$ & $7.6_{-1.1}^{+1.2}$ & $7.0_{-1.6}^{+4.1}$ & $7.59_{-0.63}^{+1.66}$ & $7.4_{-4.3}^{+7.6}$ & $\ldots$ & $7.9_{-2.4}^{+1.5}$ & $7.1_{-1.2}^{+1.9}$ \\
\hline & $5.36_{-1.23}^{+0.94}$ & $4.4_{-3.1}^{+2.2}$ & $6.31_{-0.74}^{+0.75}$ & $5.1_{-3.4}^{+1.6}$ & $0.5_{-2.6}^{+7.2}$ & $5.9_{-13.3}^{+6.1}$ & $6.66_{-1.09}^{+0.54}$ & $4.8_{-2.0}^{+1.4}$ \\
\hline \multirow[t]{2}{*}{$C_{0}$} & $23.69_{-0.21}^{+0.18}$ & $23.75_{-0.26}^{+0.28}$ & $23.58_{-0.71}^{+0.42}$ & $23.86_{-0.24}^{+0.20}$ & $23.61_{-0.54}^{+0.77}$ & $\ldots$ & $23.95_{-0.30}^{+0.38}$ & $23.56_{-0.40}^{+0.31}$ \\
\hline & $23.69_{-0.17}^{+0.15}$ & $23.79_{-0.29}^{+0.25}$ & $23.62_{-0.18}^{+0.16}$ & $23.94_{-0.27}^{+0.29}$ & $23.12_{-0.52}^{+0.84}$ & $23.83_{-0.54}^{+0.47}$ & $23.94_{-0.15}^{+0.16}$ & $23.44_{-0.31}^{+0.25}$ \\
\hline \multirow[t]{2}{*}{$\rho$} & 0.920 & 0.952 & 0.913 & 0.998 & 0.857 & $\ldots$ & 0.964 & 0.924 \\
\hline & 0.806 & 0.617 & 0.923 & 0.761 & -0.072 & 0.476 & 0.883 & 0.715 \\
\hline \multirow[t]{2}{*}{$p_{S}$} & $1.37 \mathrm{e}-14$ & $1.13 \mathrm{e}-09$ & $8.10 \mathrm{e}-07$ & $7.46 e-12$ & $1.37 \mathrm{e}-02$ & $\ldots$ & $7.32 \mathrm{e}-06$ & $1.17 \mathrm{e}-10$ \\
\hline & $2.66 \mathrm{e}-15$ & $2.20 \mathrm{e}-04$ & $1.57 \mathrm{e}-13$ & $6.11 \mathrm{e}-04$ & $8.78 \mathrm{e}-01$ & $2.33 e-01$ & $2.37 \mathrm{e}-10$ & $2.90 \mathrm{e}-06$ \\
\hline \multirow[t]{2}{*}{$N_{\text {pairs }}$} & 34 & 18 & 16 & 11 & 7 & $\ldots$ & 10 & 24 \\
\hline & 62 & 31 & 31 & 16 & 7 & 8 & 29 & 33 \\
\hline
\end{tabular}

Notes. See the notes of Table 2 . 
Table 5. Linear fits performed to the trends of Type-III galaxies in the photometric planes relating $\mu_{0, \mathrm{i}}$ and $\mu_{0, \mathrm{o}}$ with $h_{\mathrm{i}}$ and $h_{\mathrm{o}}$.

\begin{tabular}{|c|c|c|c|c|c|c|c|c|}
\hline \multicolumn{9}{|c|}{ 11) $\mu_{0, \mathrm{i}}$ vs. $\log \left(h_{\mathrm{i}}\right)$} \\
\hline & All & Spirals & S0 & $\mathrm{Sa}-\mathrm{Sab}$ & $\mathrm{Sb}-\mathrm{Sbc}$ & Sc-Scd & Barred & Unbarred \\
\hline \multirow[t]{2}{*}{$m$} & $4.3_{-1.5}^{+1.8}$ & $3.6_{-2.5}^{+3.5}$ & $5.1_{-2.1}^{+2.0}$ & $4.9_{-5.5}^{+6.8}$ & $3.7_{-4.0}^{+6.2}$ & 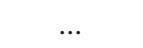 & $5.3_{-1.6}^{+2.2}$ & $4.2_{-2.5}^{+2.9}$ \\
\hline & $1.78_{-0.68}^{+0.63}$ & $1.3_{-1.2}^{+1.1}$ & $2.1_{-1.0}^{+1.0}$ & $1.3_{-2.2}^{+1.2}$ & $0.9_{-4.5}^{+3.5}$ & $-0.1_{-3.0}^{+3.7}$ & $1.99_{-1.32}^{+0.89}$ & $1.89_{-0.97}^{+1.02}$ \\
\hline \multirow[t]{2}{*}{$C_{0}$} & $18.06_{-0.58}^{+0.44}$ & $18.11_{-1.33}^{+0.99}$ & $18.15_{-0.61}^{+0.46}$ & $17.6_{-2.8}^{+2.2}$ & $18.1_{-1.7}^{+1.5}$ & $\ldots$ & $17.89_{-0.77}^{+0.57}$ & $18.20_{-1.10}^{+0.63}$ \\
\hline & $18.46_{-0.21}^{+0.23}$ & $18.49_{-0.31}^{+0.35}$ & $18.48_{-0.43}^{+0.51}$ & $18.69_{-0.24}^{+0.88}$ & $18.21_{-0.91}^{+1.50}$ & $18.62_{-0.53}^{+0.81}$ & $18.55_{-0.37}^{+0.46}$ & $18.34_{-0.36}^{+0.34}$ \\
\hline \multirow[t]{2}{*}{$\rho$} & 0.706 & 0.538 & 0.857 & 0.555 & 0.714 & $\ldots$ & 0.924 & 0.585 \\
\hline & 0.519 & 0.384 & 0.607 & 0.313 & 0.000 & -0.048 & 0.516 & 0.538 \\
\hline \multirow{2}{*}{$p_{S}$} & $5.12 \mathrm{e}-07$ & $2.14 \mathrm{e}-02$ & $6.99 \mathrm{e}-07$ & $7.67 \mathrm{e}-02$ & $7.13 e-02$ & & $8.66 \mathrm{e}-07$ & $2.67 \mathrm{e}-03$ \\
\hline & $1.57 \mathrm{e}-05$ & $3.28 \mathrm{e}-02$ & $2.92 \mathrm{e}-04$ & $2.37 \mathrm{e}-01$ & $1.00 \mathrm{e}+00$ & $9.11 \mathrm{e}-01$ & $4.14 \mathrm{e}-03$ & $1.23 \mathrm{e}-03$ \\
\hline \multirow{4}{*}{$N_{\text {pairs }}$} & 39 & 18 & 21 & 11 & 7 & $\ldots$ & 15 & 24 \\
\hline & 62 & 31 & 31 & 16 & 7 & 8 & 29 & 33 \\
\hline & & & & $\mu_{0, \mathrm{i}}$ vs. $\log$ & $\left.l_{\mathrm{i}} / R_{25}\right)$ & & & \\
\hline & All & Spirals & So & $\mathrm{Sa}-\mathrm{Sab}$ & $\mathrm{Sb}-\mathrm{Sbc}$ & Sc-Scd & Barred & Unbarred \\
\hline \multirow[t]{2}{*}{$m$} & $6.3_{-1.5}^{+1.5}$ & $5.5_{-1.9}^{+2.0}$ & $7.6_{-2.8}^{+2.0}$ & $4.7_{-1.7}^{+5.1}$ & $6.2_{-2.5}^{+1.6}$ & $\cdots$ & $6.98_{-1.76}^{+0.88}$ & $6.4_{-2.6}^{+2.4}$ \\
\hline & $4.3_{-1.6}^{+1.2}$ & $3.1_{-3.4}^{+2.9}$ & $5.77_{-0.98}^{+0.84}$ & $3.3_{-4.2}^{+2.1}$ & $-0.3_{-3.2}^{+8.0}$ & $6.5_{-7.4}^{+6.5}$ & $5.97_{-0.94}^{-0.76}$ & $4.2_{-2.8}^{+2.9}$ \\
\hline \multirow[t]{2}{*}{$C_{0}$} & $23.16_{-0.94}^{+0.95}$ & $22.7_{-1.1}^{+1.3}$ & $23.9_{-17}^{+1.2}$ & $22.3_{-1.1}^{+3.2}$ & $22.8_{-1.3}^{+1.3}$ & $\ldots$ & $23.51_{-0.95}^{+0.56}$ & $23.3_{-1.7}^{+1.5}$ \\
\hline & $21.84_{-1.10}^{+0.81}$ & $20.9_{-2.4}^{+1.8}$ & $22.89_{-0.68}^{+0.52}$ & $21.3_{-2.7}^{-1.1}$ & $18.2_{-2.2}^{-1.3 .}$ & $23.2_{-5.5}^{+4.2}$ & $23.03_{-0.64}^{+0.61}$ & $21.7_{-2.0}^{-1.1}$ \\
\hline \multirow[t]{2}{*}{$\rho$} & 0.877 & 0.889 & 0.853 & 0.945 & 0.893 & $\ldots$ & 0.899 & 0.853 \\
\hline & 0.708 & 0.444 & 0.904 & 0.458 & 0.107 & 0.524 & 0.868 & 0.599 \\
\hline \multirow[t]{2}{*}{$p_{S}$} & $2.55 \mathrm{e}-13$ & $8.39 \mathrm{e}-07$ & $9.24 \mathrm{e}-07$ & $1.12 \mathrm{e}-05$ & $6.81 \mathrm{e}-03$ & $\cdots$ & $5.18 \mathrm{e}-06$ & $1.19 \mathrm{e}-07$ \\
\hline & $1.19 \mathrm{e}-10$ & $1.24 \mathrm{e}-02$ & $3.38 \mathrm{e}-12$ & $7.46 \mathrm{e}-02$ & $8.19 \mathrm{e}-01$ & $1.83 \mathrm{e}-01$ & $1.09 \mathrm{e}-09$ & $2.33 \mathrm{e}-04$ \\
\hline \multirow{2}{*}{$N_{\text {pairs }}$} & 39 & 18 & 21 & 11 & 7 & $\ldots$ & 15 & 24 \\
\hline & 62 & 31 & 31 & 16 & 7 & 8 & 29 & 33 \\
\hline \multicolumn{9}{|c|}{ 13) $\mu_{0, \mathrm{o}}$ vs. $\log \left(h_{\mathrm{o}}\right)$} \\
\hline & All & Spirals & So & $\mathrm{Sa}-\mathrm{Sab}$ & $\mathrm{Sb}-\mathrm{Sbc}$ & Sc-Scd & Barred & Unbarred \\
\hline \multirow[t]{2}{*}{$m$} & $4.7_{-1.1}^{+1.4}$ & $5.0_{-2.7}^{+5.5}$ & $4.7_{-1.4}^{+1.5}$ & $6.2_{-5.8}^{+6.8}$ & $5.0_{-6.0}^{+10.9}$ & ... & $7.1_{-5.3}^{+4.5}$ & $4.4_{-1.4}^{+1.5}$ \\
\hline & $2.08_{-0.87}^{-0.13}$ & $1.9_{-1.5}^{+1.1}$ & $2.4_{-1.5}^{+1.4}$ & $1.8_{-2.2}^{+1.3}$ & $1.2_{-5.6}^{+0.0}$ & $1.6_{-1.8}^{+2.6}$ & $2.5_{-1.7}^{+5.3}$ & $2.0_{-1.4}^{+1.4}$ \\
\hline \multirow[t]{2}{*}{$C_{0}$} & $18.77_{-1.07}^{+0.79}$ & $18.5_{-4.2}^{+1.8}$ & $19.06_{-0.94}^{+0.88}$ & $17.4_{-5.0}^{+4.3}$ & $18.7_{-7.8}^{+2.7}$ & $\ldots$ & $17.4_{-3.4}^{+3.1}$ & $19.00_{-1.24}^{+0.92}$ \\
\hline & $19.90_{-0.44}^{+0.48}$ & $20.13_{-0.63}^{+0.74}$ & $19.48_{-0.83}^{+0.91}$ & $20.37_{-0.84}^{+1.32}$ & $20.2_{-2.2}^{+2.9}$ & $20.21_{-0.97}^{+0.77}$ & $19.80_{-0.76}^{+0.84}$ & $19.76_{-0.74}^{+0.81}$ \\
\hline \multirow[t]{2}{*}{$\rho$} & 0.713 & 0.648 & 0.835 & 0.618 & 0.571 & $\ldots$ & 0.806 & 0.731 \\
\hline & 0.414 & 0.435 & 0.432 & 0.450 & 0.321 & 0.357 & 0.432 & 0.435 \\
\hline \multirow[t]{2}{*}{$p_{S}$} & $2.24 \mathrm{e}-06$ & $3.61 \mathrm{e}-03$ & $5.64 \mathrm{e}-05$ & $4.26 \mathrm{e}-02$ & $1.80 \mathrm{e}-01$ & & $4.86 \mathrm{e}-03$ & 4.91e-05 \\
\hline & $8.13 \mathrm{e}-04$ & $1.44 \mathrm{e}-02$ & $1.52 \mathrm{e}-02$ & $8.03 \mathrm{e}-02$ & $4.82 \mathrm{e}-01$ & $3.85 \mathrm{e}-01$ & $1.94 \mathrm{e}-02$ & $1.14 \mathrm{e}-02$ \\
\hline \multirow[t]{4}{*}{$N_{\text {pairs }}$} & 34 & 18 & 16 & 11 & 7 & $\ldots$ & 10 & 24 \\
\hline & 62 & 31 & 31 & 16 & 7 & 8 & 29 & 33 \\
\hline & & & & $\iota_{0, \mathrm{o}}$ vs. $\log$ & $\left./ R_{25}\right)$ & & & \\
\hline & All & Spirals & So & $\mathrm{Sa}-\mathrm{Sab}$ & $\mathrm{Sb}-\mathrm{Sbc}$ & Sc-Scd & Barred & Unbarred \\
\hline \multirow[t]{2}{*}{$m$} & $6.60_{-0.98}^{+1.20}$ & $6.9_{-1.9}^{+2.9}$ & $6.3_{-1.4}^{+1.5}$ & $8.6_{-1.6}^{+2.0}$ & $5.9_{-2.7}^{+4.1}$ & $\ldots$ & $7.2_{-5.0}^{+3.2}$ & $6.5_{-1.1}^{+1.4}$ \\
\hline & $5.33_{-0.89}^{+0.69}$ & $4.9_{-2.4}^{+1.6}$ & $5.79_{-0.59}^{+0.71}$ & $5.6_{-1.9}^{+1.0}$ & $1.3_{-4.0}^{+7.3}$ & $5.2_{-3.8}^{+5.2}$ & $6.00_{-0.90}^{+1.30}$ & $5.32_{-1.73}^{+0.89}$ \\
\hline \multirow[t]{2}{*}{$C_{0}$} & $23.46_{-0.26}^{+0.32}$ & $23.52_{-0.48}^{+0.68}$ & $23.41_{-0.47}^{+0.55}$ & $24.03_{-0.36}^{+0.44}$ & $23.05_{-0.91}^{+1.02}$ & $\ldots$ & $23.80_{-1.42}^{+0.72}$ & $23.38_{-0.35}^{+0.44}$ \\
\hline & $23.08_{-0.41}^{+0.32}$ & $23.02_{-1.09}^{+0.41}$ & $23.10_{-0.27}^{+0.32}$ & $23.40_{-0.68}^{+0.63}$ & $21.2_{-1.4}^{+3.5}$ & $23.3_{-2.1}^{+1.9}$ & $23.52_{-0.33}^{+0.54}$ & $22.87_{-0.75}^{+0.42}$ \\
\hline \multirow[t]{2}{*}{$\rho$} & 0.888 & 0.866 & 0.909 & 0.873 & 0.821 & $\ldots$ & 0.818 & 0.928 \\
\hline & 0.804 & 0.739 & 0.848 & 0.882 & 0.000 & 0.714 & 0.905 & 0.759 \\
\hline \multirow[t]{2}{*}{$p_{S}$} & $2.55 \mathrm{e}-12$ & $3.35 \mathrm{e}-06$ & $1.10 \mathrm{e}-06$ & $4.55 \mathrm{e}-04$ & $2.34 \mathrm{e}-02$ & & $3.81 \mathrm{e}-03$ & $6.81 \mathrm{e}-11$ \\
\hline & $3.47 \mathrm{e}-15$ & $2.04 \mathrm{e}-06$ & $1.79 \mathrm{e}-09$ & $6.10 \mathrm{e}-06$ & $1.00 \mathrm{e}+00$ & $4.65 \mathrm{e}-02$ & $1.60 \mathrm{e}-11$ & $2.98 \mathrm{e}-07$ \\
\hline$N_{\text {pairs }}$ & 34 & 18 & 16 & 11 & 7 & $\ldots$ & 10 & 24 \\
\hline & 62 & 31 & 31 & 16 & 7 & 8 & 29 & 33 \\
\hline
\end{tabular}

Notes. See the notes of Table 2. 
M. C. Eliche-Moral et al.: Scaling relations of anti-truncated stellar discs in S0-Scd galaxies

Table 6. Linear fits performed to the trends of Type-III galaxies in the photometric planes relating $h_{\mathrm{i}}$ and $h_{\mathrm{o}}$.

\begin{tabular}{|c|c|c|c|c|c|c|c|c|}
\hline \multicolumn{9}{|c|}{ 15) $\log \left(h_{\mathrm{o}}\right)$ vs. $\log \left(h_{\mathrm{i}}\right)$} \\
\hline & All & Spirals & S0 & $\mathrm{Sa}-\mathrm{Sab}$ & $\mathrm{Sb}-\mathrm{Sbc}$ & Sc-Scd & Barred & Unbarred \\
\hline \multirow[t]{2}{*}{$m$} & $0.70_{-0.30}^{+0.27}$ & $0.51_{-0.72}^{+0.34}$ & $0.90_{-0.47}^{+0.59}$ & $-0.03_{-0.86}^{+0.61}$ & $0.56_{-1.05}^{+0.63}$ & & $0.25_{-0.42}^{+0.33}$ & $0.95_{-0.56}^{+0.51}$ \\
\hline & $1.018_{-0.095}^{+0.115}$ & $1.03_{-0.16}^{+0.22}$ & $1.05_{-0.16}^{+0.21}$ & $1.02_{-0.20}^{-0.28}$ & $0.69_{-0.73}^{-0.06}$ & $1.06_{-0.45}^{+0.25}$ & $1.04_{-0.20}^{+0.22}$ & $1.00_{-0.13}^{+0.17}$ \\
\hline \multirow[t]{2}{*}{$C_{0}$} & $0.488_{-0.089}^{+0.113}$ & $0.56_{-0.13}^{+0.26}$ & $0.39_{-0.15}^{+0.19}$ & $0.78_{-0.26}^{+0.34}$ & $0.49_{-0.13}^{+0.37}$ & & $0.57_{-0.11}^{+0.15}$ & $0.41_{-0.14}^{+0.23}$ \\
\hline & $0.282_{-0.035}^{+0.035}$ & $0.288_{-0.046}^{+0.049}$ & $0.239_{-0.068}^{+0.070}$ & $0.312_{-0.074}^{+0.097}$ & $0.357_{-0.056}^{+0.239}$ & $0.232_{-0.053}^{+0.120}$ & $0.242_{-0.063}^{+0.11}$ & $0.296_{-0.056}^{+0.14}$ \\
\hline \multirow[t]{2}{*}{$\rho$} & 0.463 & 0.296 & 0.717 & 0.126 & 0.321 & & 0.434 & 0.415 \\
\hline & 0.923 & 0.893 & 0.942 & 0.868 & 0.811 & 0.810 & 0.923 & 0.919 \\
\hline \multirow[t]{2}{*}{$p_{S}$} & $3.87 \mathrm{e}-03$ & $2.18 \mathrm{e}-01$ & $8.07 \mathrm{e}-04$ & $6.97 \mathrm{e}-01$ & $4.82 \mathrm{e}-01$ & & $1.38 \mathrm{e}-01$ & $4.36 \mathrm{e}-02$ \\
\hline & $1.22 \mathrm{e}-26$ & $1.39 \mathrm{e}-11$ & $2.67 \mathrm{e}-15$ & $1.33 \mathrm{e}-05$ & $2.69 \mathrm{e}-02$ & $1.49 \mathrm{e}-02$ & $9.91 \mathrm{e}-13$ & $4.31 \mathrm{e}-14$ \\
\hline \multirow{4}{*}{$N_{\text {pairs }}$} & 37 & 19 & 18 & 12 & 7 & & 13 & 24 \\
\hline & 62 & 31 & 31 & 16 & 7 & 8 & 29 & 33 \\
\hline & \multicolumn{8}{|c|}{ 16) $\log \left(h_{\mathrm{o}} / R_{25}\right)$ vs. $\log \left(h_{\mathrm{i}} / R_{25}\right)$} \\
\hline & All & Spirals & So & $\mathrm{Sa}-\mathrm{Sab}$ & $\mathrm{Sb}-\mathrm{Sbc}$ & Sc-Scd & Barred & Unbarred \\
\hline \multirow[t]{2}{*}{$m$} & $0.56_{-0.27}^{+0.28}$ & $0.58_{-0.63}^{+0.49}$ & $0.54_{-0.55}^{+0.56}$ & $0.28_{-0.75}^{+0.67}$ & $0.9_{-1.8}^{+1.0}$ & $\cdots$ & $0.28_{-0.61}^{+0.44}$ & $0.66_{-0.42}^{+0.61}$ \\
\hline & $0.93_{-0.22}^{+0.21}$ & $0.79_{-0.31}^{+0.033}$ & $1.15_{-0.37}^{+0.35}$ & $0.64_{-0.63}^{+0.53}$ & $0.87_{-0.78}^{+0.86}$ & $0.94_{-1.56}^{+0.50}$ & $1.10_{-0.40}^{+0.015}$ & $0.89_{-0.36}^{+0.29}$ \\
\hline \multirow[t]{2}{*}{$C_{0}$} & $0.13_{-0.18}^{+0.17}$ & $0.15_{-0.43}^{+0.30}$ & $0.10_{-0.30}^{+0.36}$ & $-0.03_{-0.57}^{+0.35}$ & $0.37_{-1.10}^{+0.53}$ & $\ldots$ & $-0.09_{-0.38}^{+0.28}$ & $0.20_{-0.25}^{+0.38}$ \\
\hline & $0.24_{-0.15}^{+0.15}$ & $0.15_{-0.19}^{+0.21}$ & $0.35_{-0.25}^{+0.23}$ & $0.08_{-0.35}^{+0.37}$ & $0.21_{-0.57}^{+0.44}$ & $0.20_{-1.08}^{+0.33}$ & $0.31_{-0.27}^{+0.38}$ & $0.23_{-0.23}^{+0.19}$ \\
\hline \multirow[t]{2}{*}{$\rho$} & 0.367 & 0.279 & 0.362 & 0.084 & 0.536 & $\ldots$ & 0.236 & 0.451 \\
\hline & 0.775 & 0.657 & 0.865 & 0.506 & 0.643 & 0.619 & 0.856 & 0.716 \\
\hline \multirow[t]{2}{*}{$p_{S}$} & $2.54 \mathrm{e}-02$ & $2.47 \mathrm{e}-01$ & $1.40 \mathrm{e}-01$ & $7.95 \mathrm{e}-01$ & $2.15 \mathrm{e}-01$ & & $4.37 \mathrm{e}-01$ & $2.69 \mathrm{e}-02$ \\
\hline & $1.52 \mathrm{e}-13$ & $5.98 \mathrm{e}-05$ & $3.36 \mathrm{e}-10$ & $4.56 \mathrm{e}-02$ & $1.19 \mathrm{e}-01$ & $1.02 \mathrm{e}-01$ & $3.32 \mathrm{e}-09$ & $2.86 \mathrm{e}-06$ \\
\hline \multirow{2}{*}{$N_{\text {pairs }}$} & 37 & 19 & 18 & 12 & 7 & $\ldots$ & 13 & 24 \\
\hline & 62 & 31 & 31 & & & & & \\
\hline
\end{tabular}

Notes. See the notes of Table 2. 
A\&A 580, A33 (2015)

Table 7. Linear fits performed to the trends of Type-III galaxies in the photometric planes relating $R_{25}$ with $h_{\mathrm{i}}, h_{\mathrm{o}}$, and $R_{\text {brkIII- }}$.

\begin{tabular}{|c|c|c|c|c|c|c|c|c|}
\hline \multicolumn{9}{|c|}{ 17) $\log \left(R_{25}\right)$ vs. $\log \left(h_{\mathrm{i}}\right)$} \\
\hline & All & Spirals & So & $\mathrm{Sa}-\mathrm{Sab}$ & $\mathrm{Sb}-\mathrm{Sbc}$ & Sc-Scd & Barred & Unbarred \\
\hline$m$ & $\begin{array}{l}0.47_{-0.21}^{+0.19} \\
0.84_{-0.11}^{+0.12}\end{array}$ & $\begin{array}{l}0.50_{-0.55}^{+0.37} \\
0.96_{-0.20}^{+0.21}\end{array}$ & $\begin{array}{l}0.42_{-0.16}^{+0.20} \\
0.70_{-0.15}^{+0.16}\end{array}$ & $\begin{array}{l}0.00_{-0.83}^{+0.64} \\
0.92_{-0.24}^{+0.29}\end{array}$ & $\begin{array}{c}0.60_{-0.90}^{+0.52} \\
1.49_{-0.54}^{+0.73}\end{array}$ & $\begin{array}{c}\cdots \\
0.87_{-0.46}^{+0.31}\end{array}$ & $\begin{array}{l}0.25_{-0.30}^{+0.23} \\
0.76_{-0.15}^{+0.20}\end{array}$ & $\begin{array}{l}0.56_{-0.33}^{+0.29} \\
0.82_{-0.17}^{+0.20}\end{array}$ \\
\hline$C_{0}$ & $\begin{array}{l}0.771_{-0.065}^{+0.079} \\
0.705_{-0.041}^{+0.040}\end{array}$ & $\begin{array}{c}0.79_{-0.14}^{+0.20} \\
0.682_{-0.067}^{+0.061}\end{array}$ & $\begin{array}{l}0.734_{-0.048}^{+0.057} \\
0.740_{-0.074}^{+0.073}\end{array}$ & $\begin{array}{c}0.99_{-0.23}^{+0.34} \\
0.681_{-0.099}^{+0.083}\end{array}$ & $\begin{array}{l}0.73_{-0.19}^{+0.26} \\
0.59_{-0.17}^{+0.19}\end{array}$ & $\begin{array}{c}\ldots \\
0.707_{-0.082}^{+0.093}\end{array}$ & $\begin{array}{l}0.812_{-0.083}^{+0.100} \\
0.725_{-0.072}^{+0.067}\end{array}$ & $\begin{array}{l}0.726_{-0.087}^{+0.140} \\
0.706_{-0.065}^{+0.071}\end{array}$ \\
\hline$\rho$ & $\begin{array}{l}0.530 \\
0.859\end{array}$ & $\begin{array}{l}0.335 \\
0.852\end{array}$ & $\begin{array}{l}0.696 \\
0.843\end{array}$ & $\begin{array}{c}-0.014 \\
0.859\end{array}$ & $\begin{array}{l}0.571 \\
0.901\end{array}$ & $\begin{array}{c}\ldots \\
0.881\end{array}$ & $\begin{array}{l}0.418 \\
0.849\end{array}$ & $\begin{array}{l}0.528 \\
0.855\end{array}$ \\
\hline$p_{S}$ & $\begin{array}{l}4.41 \mathrm{e}-04 \\
4.07 \mathrm{e}-19\end{array}$ & $\begin{array}{l}1.61 \mathrm{e}-01 \\
1.22 \mathrm{e}-09\end{array}$ & $\begin{array}{l}4.57 \mathrm{e}-04 \\
2.64 \mathrm{e}-09\end{array}$ & $\begin{array}{l}9.66 \mathrm{e}-01 \\
2.05 \mathrm{e}-05\end{array}$ & $\begin{array}{l}1.80 \mathrm{e}-01 \\
5.62 \mathrm{e}-03\end{array}$ & $\begin{array}{c}\ldots \\
3.85 \mathrm{e}-03\end{array}$ & $\begin{array}{l}1.07 \mathrm{e}-01 \\
5.87 \mathrm{e}-09\end{array}$ & $\begin{array}{l}7.95 \mathrm{e}-03 \\
2.28 \mathrm{e}-10\end{array}$ \\
\hline$N_{\text {pairs }}$ & $\begin{array}{l}40 \\
62\end{array}$ & $\begin{array}{l}19 \\
31\end{array}$ & $\begin{array}{l}21 \\
31\end{array}$ & $\begin{array}{l}12 \\
16\end{array}$ & $\begin{array}{l}7 \\
7\end{array}$ & $\begin{array}{l}\cdots \\
8\end{array}$ & $\begin{array}{l}16 \\
29\end{array}$ & $\begin{array}{l}24 \\
33\end{array}$ \\
\hline \multicolumn{9}{|c|}{ 18) $\log \left(R_{25}\right)$ vs. $\log \left(h_{\mathrm{o}}\right)$} \\
\hline & All & Spirals & S0 & $\mathrm{Sa}-\mathrm{Sab}$ & $\mathrm{Sb}-\mathrm{Sbc}$ & Sc-Scd & Barred & Unbarred \\
\hline$m$ & $\begin{array}{l}0.33_{-0.17}^{+0.16} \\
0.68_{-0.13}^{+0.16}\end{array}$ & $\begin{array}{l}0.34_{-0.65}^{+0.40} \\
0.77_{-0.21}^{+0.29}\end{array}$ & $\begin{array}{l}0.29_{-0.15}^{+0.11} \\
0.56_{-0.19}^{+0.24}\end{array}$ & $\begin{array}{l}0.19_{-0.59}^{+0.55} \\
0.74_{-0.25}^{+0.36}\end{array}$ & $\begin{array}{l}0.17_{-1.40}^{+0.97} \\
1.75_{-0.98}^{+0.88}\end{array}$ & $\begin{array}{c}\ldots \\
0.72_{-0.43}^{+0.36}\end{array}$ & $\begin{array}{l}0.14_{-0.32}^{+0.46} \\
0.58_{-0.21}^{+0.30}\end{array}$ & $\begin{array}{l}0.34_{-0.18}^{+0.18} \\
0.71_{-0.20}^{+0.26}\end{array}$ \\
\hline$C_{0}$ & $\begin{array}{c}0.69_{-0.11}^{+0.13} \\
0.554_{-0.088}^{+0.080}\end{array}$ & $\begin{array}{l}0.69_{-0.28}^{+0.47} \\
0.50_{-0.16}^{+0.13}\end{array}$ & $\begin{array}{c}0.672_{-0.076}^{+0.108} \\
0.64_{-0.14}^{+0.13}\end{array}$ & $\begin{array}{c}0.84_{-0.40}^{+0.44} \\
0.50_{-0.21}^{+0.17}\end{array}$ & $\begin{array}{l}0.71_{-0.43}^{+0.96} \\
0.01_{-0.37}^{+0.58}\end{array}$ & $\begin{array}{c}\ldots \\
0.56_{-0.19}^{+0.17}\end{array}$ & $\begin{array}{c}0.81_{-0.30}^{+0.24} \\
0.62_{-0.15}^{+0.13}\end{array}$ & $\begin{array}{l}0.66_{-0.13}^{+0.15} \\
0.53_{-0.15}^{+0.13}\end{array}$ \\
\hline$\rho$ & $\begin{array}{l}0.479 \\
0.841\end{array}$ & $\begin{array}{l}0.182 \\
0.859\end{array}$ & $\begin{array}{l}0.734 \\
0.802\end{array}$ & $\begin{array}{l}0.203 \\
0.876\end{array}$ & $\begin{array}{l}0.036 \\
0.964\end{array}$ & $\begin{array}{c}\ldots \\
0.810\end{array}$ & $\begin{array}{l}0.115 \\
0.796\end{array}$ & $\begin{array}{l}0.536 \\
0.864\end{array}$ \\
\hline$p_{S}$ & $\begin{array}{l}2.73 \mathrm{e}-03 \\
1.23 \mathrm{e}-17\end{array}$ & $\begin{array}{l}4.55 \mathrm{e}-01 \\
6.34 \mathrm{e}-10\end{array}$ & $\begin{array}{l}5.28 \mathrm{e}-04 \\
5.82 \mathrm{e}-08\end{array}$ & $\begin{array}{l}5.27 \mathrm{e}-01 \\
8.44 \mathrm{e}-06\end{array}$ & $\begin{array}{l}9.39 \mathrm{e}-01 \\
4.54 \mathrm{e}-04\end{array}$ & $\begin{array}{c}\ldots \\
1.49 \mathrm{e}-02\end{array}$ & $\begin{array}{l}7.07 \mathrm{e}-01 \\
2.38 \mathrm{e}-07\end{array}$ & $\begin{array}{l}6.98 \mathrm{e}-03 \\
9.44 \mathrm{e}-11\end{array}$ \\
\hline$N_{\text {pairs }}$ & $\begin{array}{l}37 \\
62\end{array}$ & $\begin{array}{l}19 \\
31\end{array}$ & $\begin{array}{l}18 \\
31\end{array}$ & $\begin{array}{l}12 \\
16\end{array}$ & $\begin{array}{l}7 \\
7\end{array}$ & $\begin{array}{l}\cdots \\
8\end{array}$ & $\begin{array}{l}13 \\
29\end{array}$ & $\begin{array}{l}24 \\
33\end{array}$ \\
\hline \multicolumn{9}{|c|}{ 19) $\log \left(R_{25}\right)$ vs. $\log \left(R_{\text {brkIII }}\right)$} \\
\hline & All & Spirals & S0 & $\mathrm{Sa}-\mathrm{Sab}$ & $\mathrm{Sb}-\mathrm{Sbc}$ & Sc-Scd & Barred & Unbarred \\
\hline$m$ & $\begin{array}{l}0.26_{-0.18}^{+0.20} \\
0.82_{-0.13}^{+0.12}\end{array}$ & $\begin{array}{l}0.17_{-0.38}^{+0.51} \\
0.92_{-0.18}^{+0.21}\end{array}$ & $\begin{array}{l}0.36_{-0.22}^{+0.23} \\
0.66_{-0.22}^{+0.20}\end{array}$ & $\begin{array}{c}-0.19_{-0.23}^{+0.34} \\
0.87_{-0.30}^{+0.30}\end{array}$ & $\begin{array}{l}0.45_{-0.80}^{+1.53} \\
1.34_{-0.47}^{+1.38}\end{array}$ & $\begin{array}{c}\ldots \\
0.82_{-0.32}^{+0.30}\end{array}$ & $\begin{array}{l}0.19_{-0.29}^{+0.30} \\
0.68_{-0.23}^{+0.25}\end{array}$ & $\begin{array}{l}0.33_{-0.27}^{+0.31} \\
0.84_{-0.21}^{+0.19}\end{array}$ \\
\hline$C_{0}$ & $\begin{array}{l}0.67_{-0.20}^{+0.19} \\
0.22_{-0.13}^{+0.13}\end{array}$ & $\begin{array}{c}0.78_{-0.48}^{+0.39} \\
0.09_{-0.20}^{+0.18}\end{array}$ & $\begin{array}{c}0.53_{-0.23}^{+0.20} \\
0.39_{-0.20}^{+0.23}\end{array}$ & $\begin{array}{l}1.16_{-0.31}^{+0.26} \\
0.15_{-0.33}^{+0.34}\end{array}$ & $\begin{array}{c}0.48_{-1.46}^{+0.65} \\
-0.24_{-1.16}^{+0.46}\end{array}$ & $\begin{array}{c}\ldots \\
0.15_{-0.23}^{+0.27}\end{array}$ & $\begin{array}{l}0.71_{-0.29}^{+0.28} \\
0.32_{-0.25}^{+0.24}\end{array}$ & $\begin{array}{c}0.60_{-0.31}^{+0.29} \\
0.22_{-0.19}^{+0.21}\end{array}$ \\
\hline$\rho$ & $\begin{array}{l}0.404 \\
0.735\end{array}$ & $\begin{array}{l}0.018 \\
0.758\end{array}$ & $\begin{array}{l}0.726 \\
0.708\end{array}$ & $\begin{array}{c}-0.315 \\
0.691\end{array}$ & $\begin{array}{l}0.429 \\
0.786\end{array}$ & $\begin{array}{c}\ldots \\
0.857\end{array}$ & $\begin{array}{l}0.294 \\
0.742\end{array}$ & $\begin{array}{l}0.352 \\
0.752\end{array}$ \\
\hline$p_{S}$ & $\begin{array}{l}9.67 \mathrm{e}-03 \\
1.09 \mathrm{e}-11\end{array}$ & $\begin{array}{l}9.43 \mathrm{e}-01 \\
7.84 \mathrm{e}-07\end{array}$ & $\begin{array}{l}1.95 \mathrm{e}-04 \\
8.50 \mathrm{e}-06\end{array}$ & $\begin{array}{l}3.19 \mathrm{e}-01 \\
3.02 \mathrm{e}-03\end{array}$ & $\begin{array}{l}3.37 \mathrm{e}-01 \\
3.62 \mathrm{e}-02\end{array}$ & $\begin{array}{c}\cdots \\
6.53 \mathrm{e}-03\end{array}$ & $\begin{array}{l}2.69 \mathrm{e}-01 \\
4.14 \mathrm{e}-06\end{array}$ & $\begin{array}{l}9.15 \mathrm{e}-02 \\
4.61 \mathrm{e}-07\end{array}$ \\
\hline$N_{\text {pairs }}$ & $\begin{array}{l}40 \\
62 \\
\end{array}$ & $\begin{array}{l}19 \\
31 \\
\end{array}$ & $\begin{array}{l}21 \\
31 \\
\end{array}$ & $\begin{array}{l}12 \\
16 \\
\end{array}$ & $\begin{array}{l}7 \\
7 \\
\end{array}$ & $\begin{array}{l}\cdots \\
8 \\
\end{array}$ & $\begin{array}{l}16 \\
29 \\
\end{array}$ & $\begin{array}{l}24 \\
33 \\
\end{array}$ \\
\hline
\end{tabular}

Notes. See the notes of Table 2 . 